\title{
Phytocenotic features of Calluna vulgaris (L.) Hill. in Ukrainian Polesie
}

\author{
Oleksandr Lukash ${ }^{1 *}$, Viktor Melnyk², Hanna Danko', Oleksandr Rak², \\ Yurii Karpenko ${ }^{1}$, Petro Buzunko ${ }^{1}$ \\ 'Department of Ecology and Nature Conservation, T. H. Shevchenko National University "Chernihiv Collehium", \\ Hetman Polubotko Str., 53, 14013, Chernihiv, Ukraine \\ ${ }^{2}$ Department of Natural flora, M.M. Gryshko National Botanical Garden of the National Academy \\ of Sciences of Ukraine, Timiriazievska Str., 1, 01014, Kyiv, Ukraine \\ "corresponding author's e-mail: lukash2011@ukr.net
}

Received: 21 March 2020 / Accepted: 8 May 2020

The article is dedicated to the memory of the tireless researcher of the Ukrainian Polesie flora and vegetation Professor Tetiana Andrienko (1938-2016)

\begin{abstract}
Middle-aged pine and birch-pine forests of the Dicrano-Pinenion suballiance are the most common biotopes of Calluna vulgaris (L.) Hill. in Ukrainian Polesie. This species occurs mostly as solitary plants in forest phytocenoses; rarely its projective coverage can reach $40-45 \%$. C. vulgaris has the highest constancy in phytocenoses of the Peucedano-Pinetum association in Rightbank Polesie. The forests of the Piceo-Vaccinienion uliginosi Seibert in Oberd. (ed.) 1992 suballiance and the Piceion excelsae Pawłowski et al. 1928 alliance are rare habitats of C. vulgaris; they occur only in Right-bank Polesie of Ukraine. Heathlands are rare ecosystems for Ukrainian Polesie, their vegetation is represented by plant communities of the Calluno-Ulicetea Br.-B1. et Tx. ex Klika et Hadač 1944 and the Nardetea strictae Rivas Goday et Borja Carbonell in Rivas Goday et Mayor Lopez 1966 classes. They are forming in the significantly anthropogenically disrupted territories (e.g. areas after wildfires) or in the areas with intensive human activities, firstly in the military training areas. The phytocenoses of the Calluno-Genistetum pilosae R. Tx. 1937 association occur only in Right-bank Polesie and are the largest heathlands of Ukrainian Polesie in general. For example, in the Rivne region, their area is up to $20 \mathrm{~km}^{2}$. The area of heathlands, belonging to the Calluno-Nardetum strictae Hrync. 1959 association, does not exceed several hectares. The phytocenoses of the Scabioso canescentis-Genistetum Balcerk. et Brzeg 1993 association are represented with small plots in landscapes of pine forests and less frequently in mixed forests on upland terraces of Left-bank Polesie rivers. The characteristic feature of Calluna vulgaris spreading in Right-bank Polesie of Ukraine is its large participation (sometimes with the projective cover up to $80 \%)$ and high constancy $(92,7 \%)$ in phytocenoses of oligotrophic bogs of the Ledo-Sphagnetum magellanici (Malc. 1929) Kästner et Flössner 1933 association. The populations of Calluna vulgaris, explored in the forest phytocenoses of the Cladonio-Pinetum Juraszek 1927 association on the territory of Ukrainian Polesie, are incomplete. Their age spectrum maximum falls on generative individuals. The "Right-bank" population of Calluna vulgaris are characterized by more active proliferation of plants and spreading on new territories. Aging processes dominate among the "left-bank" populations. Generally, environmental conditions of Ukrainian Right-bank Polesie are more favorable to Calluna vulgaris, than conditions of Left-bank Polesie.
\end{abstract}

Keywords: Calluna vulgaris (L.) Hill., Polesie, plant communities, syntaxonomy, population. 


\section{Introduction}

Calluna vulgaris (L.) Hill., European Boreo-temperate element of flora, has a large geographic distribution and is dominating in many heath ecosystems. Such a type of vegetation prevails in many heathlands of northern and western Europe. In Ukrainian Polesie Calluna vulgaris is at the southern border of its distribution. That is what causes the affect on the species distribution in the region. The purpose of our study was to investigate the cenotic features of the Calluna vulgaris habitats and its population structure in Ukrainian Polesie.

Heathlands are highly dynamic habitats, with strong biotic and abiotic interactions affected by external drivers, despite their appearance as a homogeneous, stable ecosystem. All of the factors studied influence diversity patterns, community structure and general quality of the habitat (Fagúndez, 2013). As experience of European (in particular, Polish) scientists shows (Adamska et al., 2015), study of main heath formations and accompanying plant communities is very important for management, treatment and also conservation of the Calluna vulgaris ecological systems.

\section{Study area}

Ukrainian Polesie is the southern part of the Polessian lowland within Ukraine (Fig. 1). Poorly defined relief, considerable distribution of water-ice deposits of sand, and high marshiness are characteristic features of Polesie land- scapes. Poor sandy soils and marshiness favored the conservation of natural vegetation, which occupies here about $2 / 3$ of the area (Andrienko et al., 2006). During the maximum glaciations most of Ukrainian Polesie was covered by glacier. In modern relief glacial forms are most noticeable in the west of Polesie.

The Dnieper is the main waterway of Ukrainian Polesie. The Dnieper divides Ukrainian Polesie into Rightbank (Fig.1: 1a) and Left-bank Polesie (Fig.1: 1b). There are no significant differences between Right-bank Polesie and Left-Bank Polesie in the amount of weather elements. However, Left-bank Polesie has more continental climate in comparison with Right-bank Polesie. Karst processes are observed in some regions of Right-bank Polesie. So-called loess "islands" are available in Left-bank Polesie, where erosion processes take place (Lukash et al., 2018, 2019). Soils of Polesie are highly pattern structured. Rise of the ground level by only a few decimeters in Polesie usually causes major changes in soil-forming processes and vegetation. Sod-podzolic soils are most common. They occupy about $70 \%$ of the territory of Ukrainian Polesie. The sod-podzolic soils vary greatly in mechanical composition, humidity and trophicity. Sandy-loam and clayish sandyloam soils dominate by mechanical composition. Both pine and deciduous forests are forming on those types of soils. Gray forest soils are common on loamy loessial sediments, mainly in Left-bank Polesie.

According to various literature sources, forest areas occupy $26-29 \%$ of the territory of Ukrainian Polesie (Andrienko et al., 2006). The considerable distribution of sand deposits in Ukrainian Polesie causes large areas of pine forests. Climatic conditions of Polesie are favorable for the

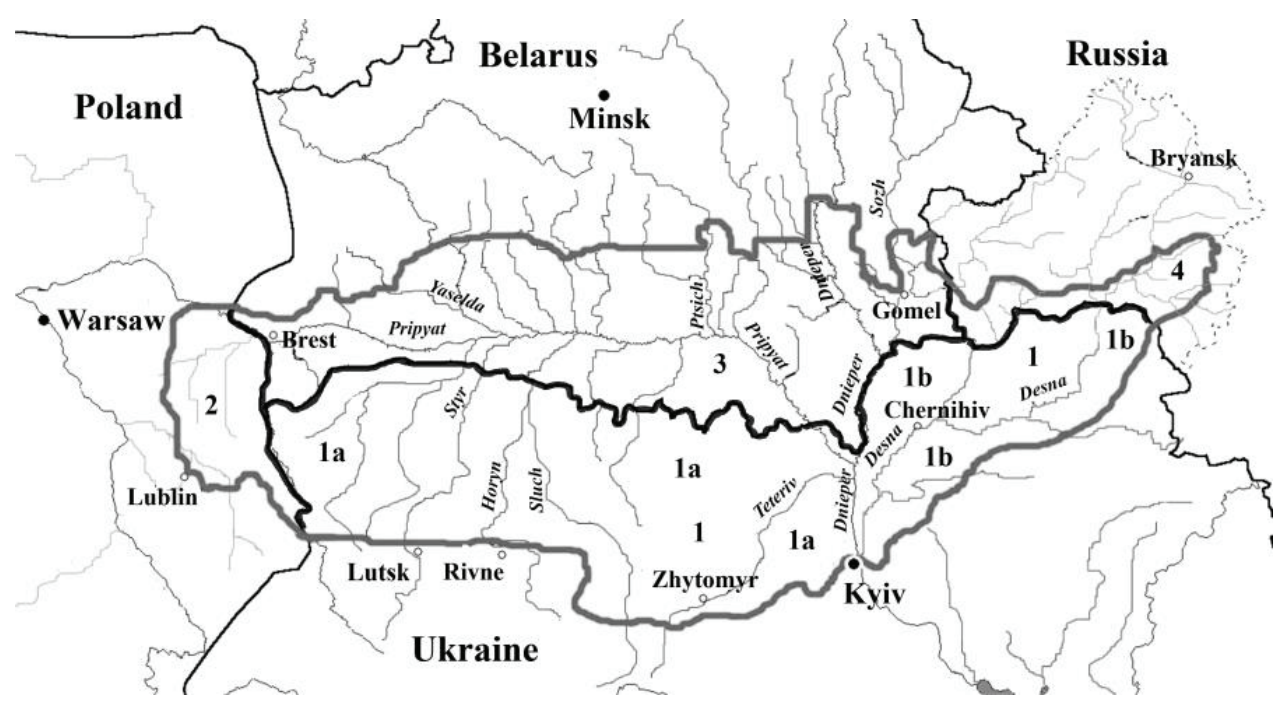

Figure 1. Schematic map of Polesie. Map symbols: 1 - Ukrainian Polesie (1a - Right-bank, 1b- Leftbank), 2 - Lublin Polesie, 3 - Belarusian Polesie, 4 - Nerissa and Desna Polesie 
growth of deciduous forests, which are local climax zonal communities. However, there are differences in distribution of deciduous forests between Right-bank and Leftbank Polesie. For example, the areal of the Carpinion betuli Issler 1931 alliance forests includes all Right-bank Polesie and only part of Left-bank Polesie (within the boundaries of the Chernihiv region) (Andrienko et al., 2006).

A high groundwater level and its slow motion favor spreading of wetland vegetation here. The climate conditions of Polesie contributed to the development of mires and in combination with mineral deficiency of the surface formations, have led to sphagnum mires formation (Fijałkowski et al., 2002). However, Left-bank Polesie is characterized by less quantity of bogs. In addition, Leftbank Polesie, compared to Right-bank Polesie, was more affected by the drainage reclamation in 1970s-1980s.

Obviously, the regularities of Calluna vulgaris distribution in these two regions of Ukrainian Polesie were affected by some differences in natural conditions (including vegetation cover) of Right-bank and Left-bank Polesie.

\section{Material and methods}

The materials for the article were collected during the field research of the Polesie vegetation in 1995-2019. The field study of the vegetation was carried out by geobotanical methods (Korchahin \& Lavrenko, 2012). The vegetation descriptions were taken during the optimum of vegetation period in the areas of 400-625 $\mathrm{m}^{2}$ (in forest phytocenoses) and 100-125 $\mathrm{m}^{2}$ (in herb and dwarf shrub phytocenoses). The projective coverage of plant communities' layers and coverage of all the species was noted. Cover abundance scale is the following: +- up to $1 \%, 1-1-5 \%, 2-6-$ $15 \%, 3-16-25 \%, 4-26-50 \%, 5>50 \%$. The tables of the floristic structure of the plant communities for Calluna vulgaris include the percentage share.

366 phytosociological authors relevés were done. 297 geobotanical relevés from Professor Tatiana Andrienko's field books 1972-1985, which had been transferred to the first author of this article during her lifetime, were analyzed. 30 published geobotanical relevés, taken on the territory of Desniansko-Starogutskyi National Nature Park, were also used (Panchenko, 2013). In total, 693 relevés were analyzed.

The documentation has been compiled separately for Right-bank Polesie (total number of relevés 391 including 247 relevés with Calluna vulgaris) and Left-bank Polesie (total number of relevés 302 including 195 relevés with Calluna vulgaris). Phytocenotic tables include the relevés with the projective coverage of Calluna vulgaris equal to or more than $1 \%$.

Syntaxa were identified according to W. Matuszkiewicz (2019), Chojnacka et al. (2010a, 2010b), Bulokhov \& So- lomeshch (2003). Syntax names are ordered according to Mucina et al. (2016).

The field populations studies were carried out according to Rabotnov (1964). The Calluna vulgaris ontogenetic stages and evaluation indexes of the general ontogenetic status of populations (Index innovation $\left(I_{i n n}\right)$, Index senilis $\left(I_{\text {sen }}\right)$, Index generative $\left(I_{\text {gen }}\right)$, Index aetas $\left.\left(I_{e}\right)\right)$ were determined according to Kovalenko (2015). The evaluation indexes of the general ontogenetic status of the populations are measured in per cent and defined as ratio of individuals quantity in different ontogenetic stages to the total quantity of plants: seedlings (p), juvenile (j), immature (im) and virginile (v) individuals $-I_{i n n}$; old generative individuals $\left(\mathrm{g}_{3}\right)$ and senile plants $(\mathrm{s})-I_{\text {sen }}$; young generative $\left(\mathrm{g}_{1}\right)$ and mature generative individuals $\left(\mathrm{g}_{2}\right)-I_{\text {gen }}$. Index aetas is the ratio of Index senilis to Index innovation: $I_{\text {aet }}=I_{\text {sen }} / I_{\text {inn }}$

\section{Results and discussion}

In Ukrainian Polesie Calluna vulgaris grows mainly in the intrazonal boreal-temperate grasslands and heath plant communities, in swamps and also in pine, mixed and spruce forests. The general scheme of the Ukrainian Polesie plant communities with Calluna vulgaris is the following (note: * - only in Right-bank Polesie, ** - only in Left-bank Polesie):

Class: Calluno-Ulicetea Br.-B1. et Tx. ex Klika et Hadač 1944

Order: Vaccinio myrtilli-Genistetalia pilosae Schubert ex Passarge 1964

Alliance: Calluno-Genistion pilosae P. Duvigneaud 1944

*Association: Calluno-Genistetum pilosae R.Tx. 1937

Alliance: Calluno-Arctostaphylion uvae-ursi Tx. et Preising in Preising 1949

**Association: Scabioso canescentis-Genistetum Balcerk. et Brzeg 1993

Class: Nardetea strictae Rivas Goday et Borja Carbonell in Rivas Goday et Mayor López 1966

Order: Nardetalia strictae Preising 1950

Boreo-temperate group of alliances

Alliance: Violion caninae Schwickerath 1944

Association: Calluno-Nardetum strictae Hrync. 1959

Class: Oxycocco-Sphagnetea Br.-Bl. et Tx. ex Westhoff et al. 1946

Order: Sphagnetalia medii Kästner et Flössner 1933

Alliance: Sphagnion medii Kästner et Flössner 1933

*Association: Ledo-Sphagnetum magellanici (Malc. 1929)

Kästner et Flössner 1933

Class:Vaccinio-Piceetea Br.-B1. in Br.-B1. et al. 1939

Order: Pinetalia sylvestris Oberd. 1957

Alliance: Dicrano-Pinion sylvestris (Libbert 1933) W. Matuszkiewicz 1962 
Suballiance: Dicrano-Pinenion Seibert in Oberd. (ed.) 1992

Association: Cladonio-Pinetum Juraszek 1927

Association: Peucedano-Pinetum W. Matuszkiewicz (1962) 1973

**Association: Veronico incanae-Pinetum Bulokhov et Solomeshch 2003

Association: Molinio-Pinetum W. Matuszkiewicz et J. Matuszkiewicz 1973

Association: Querco-Piceetum (W. Matuszkiewicz 1952) W. Matuszkiewicz et Polak 1955

Suballiance: Piceo-Vaccinienion uliginosi Seibert in Oberd. (ed.) 1992

*Association: Vaccinio uliginosi-Pinetum Kleist 1929

Order: Piceetalia excelsae Pawłowski et al. 1928

Alliance: Piceion excelsae Pawłowski et al. 1928

*Association: Sphagno girgensohnii-Piceetum Polak 1962

The intrazonal boreo-temperate heath vegetation is represented by plant communities of the Calluno-Ulicetea (Table 1) and the Calluno-Nardetum strictae classes (Table 2).

Relevés 1-3 (Table 1) belong to the Calluno-Genistetum pilosae association (the Calluno-Ulicetea pilosae class). The cenoses of the Calluno-Genistetum pilosae association, which are present only in Right-bank Polesie, occupy the largest areas (up to $20 \mathrm{~km}^{2}$ ). Calluno-Genistetum pilosae encompasses dry heaths represented, as well as in Poland (Adamska et al., 2015), by the anthropogenic communities, developing on poor sandy soils, as a degeneration phase of pine and mixed forests.

The heathlands of the Calluno-Genistetum pilosae association are common on large territories of the artillery shooting range in Hoshchansk, Kostopil and Rivne districts of the Rivne region and on the northern outskirts of Volodymyr-Volynskyi (the Volyn region). A shrub layer is formed with Sarothamnus scoparius (L.) W.D.J. Koch (the projective cover of 40-60\%). Calluna vulgaris dominates in a herb layer and has the projective cover of 50$80 \%$. Sarothamnus scoparius is a diagnostic species of the Calluno-Genistetum pilosae association and the CallunoGenistion pilosae alliance. Moss layer is formed with Pleurozium schreberi (Brid.) Mitt. Polessian communities with Sarothamnus scoparius differ in composition from the plant communities, described in Poland near the city of Torun. The community dominated by common groom Sarothamnus scoparius covers the areas adjacent to heathlands Pohlio-Callunetum Shimwell 1973 em. Brzeg 1981 and Arctostaphylo-Callunetum R.Tx. et Prsg 1940, as well as with grasslands of the gray clubawn grass Spergulo vernalis-Corynephoretum (R.Tx. 1928) Libb. 1933 (Chojnacka et al., 2010a).

The low-altitude heaths of the Atlantic and subcontinental regions of temperate Europe, belonging to the Scabioso canescentis-Genistetum association (Table 1, relevés 4-8), are represented only in Left-bank Polesie. They are rare and occupy small areas (up to $200 \mathrm{~m}^{2}$ ) among pine forests on the upland river terraces. The characteristic ( $\mathrm{Ge}$ nista tinctoria L., Scabiosa ochroleuca L.) and differential (Polygonatum odoratum (Mill.) Druce, Solidago virgaurea L., Vincetoxicum hirundinaria Medik.) species of the association are present in all the described phytocenoses. Calluna vulgaris grows in curtains; its projective coverage does not exceed $10 \%$. The plant communities have a moss layer (with the projective cover of 25-90\%). Pleurozium schreberi dominates in the layer.

The heathlands of Ukrainian Polesie are represented by the plant comunities of the Calluno-Nardetum strictae association (the Nardetea strictae class). They occupy the

Table 1. Floristic structure of the Ukrainian Polesie heath plant communities of the Calluno-Ulicetea class: the Calluno-Genistetum pilosae (relevés 1-3) and the Scabioso canescentis-Genistetum (relevés 4-8) associations

\begin{tabular}{|c|c|c|c|c|c|c|c|c|}
\hline \multirow{2}{*}{$\frac{\text { Region }}{\text { Relevé number }}$} & \multicolumn{3}{|c|}{ Right-bank Polesie } & \multicolumn{5}{|c|}{ Left-bank Polesie } \\
\hline & 1 & 2 & 3 & 4 & 5 & 6 & 7 & 8 \\
\hline Shrub layer (b) cover $[\%]$ & 60 & 40 & 5 & 1 & 5 & $<1$ & 0 & 15 \\
\hline Herb and dwarf shrub layer (c) cover [\%] & 80 & 50 & 90 & 40 & 35 & 45 & 50 & 25 \\
\hline Mosses layer $(\mathrm{d})$ cover $[\%]$ & 10 & 0 & 30 & 25 & 70 & 80 & 90 & 85 \\
\hline Lichens layer (e) cover [\%] & 0 & 0 & 0 & 0 & 10 & 5 & 0 & 5 \\
\hline \multicolumn{9}{|l|}{$\begin{array}{l}\text { Ch., D. Ass. Calluno-Genistetum pilosae } \\
\text { and All. Calluno-Genistion pilosae }\end{array}$} \\
\hline Sarothamus scoparius & 5 & 4 & + & . & . & & . & . \\
\hline Genista germanica & + & + & + & . & . & . & . & . \\
\hline
\end{tabular}




\begin{tabular}{|c|c|c|c|c|c|c|c|c|}
\hline \multirow{2}{*}{$\frac{\text { Region }}{\text { Relevé number }}$} & \multicolumn{3}{|c|}{ Right-bank Polesie } & \multicolumn{5}{|c|}{ Left-bank Polesie } \\
\hline & 1 & 2 & 3 & 4 & 5 & 6 & 7 & 8 \\
\hline \multicolumn{9}{|c|}{ Ch., D. Ass. Scabioso canescentis-Genistetum } \\
\hline Genista tinctoria & . & . & . & + & + & 1 & + & + \\
\hline Polygonatum odoratum & . & . & . & + & 1 & + & + & + \\
\hline Scabiosa ochroleuca & . & . & . & + & + & + & + & + \\
\hline Solidago virgaurea & . & . & . & + & + & + & + & + \\
\hline Vincetoxicum hirundinaria & . & . & . & + & + & + & + & + \\
\hline \multicolumn{9}{|l|}{ Ch. All. Calluno-Arctostaphylion } \\
\hline Arctostaphylos uva-ursi & . & . & . & . & 1 & 1 & + & + \\
\hline Carex praecox & . & . & . & + & + & + & . & . \\
\hline Peucedanum oreoselinum & . & . & . & + & + & 1 & 2 & + \\
\hline Scorzonera humilis & . & . & . & . & . & + & + & + \\
\hline \multicolumn{9}{|l|}{$\begin{array}{l}\text { Ch. Cl. Calluno-Ulicetea } \\
\text { and O. Vaccinio myrtilli-Genistetalia pilosae }\end{array}$} \\
\hline Calluna vulgaris & 80 & 50 & 30 & 10 & 5 & 8 & 10 & 5 \\
\hline Carex ericetorum & + & + & + & + & 1 & 1 & + & . \\
\hline Dicranum scoparium & . & . & . & . & + & 2 & 1 & + \\
\hline Hieracium umbellatum & + & + & + & + & + & + & + & + \\
\hline Vaccinium myrtillus & 1 & + & 1 & . & . & . & . & . \\
\hline \multicolumn{9}{|l|}{ Accompanying species } \\
\hline \multicolumn{9}{|l|}{ Cl. Vaccinio-Piceetea } \\
\hline Ajuga reptans & . & . & + & . & . & . & . & . \\
\hline Cladina rangiferina & . & . & . & . & 1 & 1 & . & . \\
\hline Cladonia uncialis & . & . & . & . & 1 & + & . & 1 \\
\hline Dicranum rugosum & . & . & . & . & . & . & . & 4 \\
\hline Luzula pilosa & . & . & 1 & . & . & . & . & . \\
\hline Melampyrum pratense & . & . & . & + & . & . & . & . \\
\hline Pinus sylvestris (b) & 1 & 1 & 1 & 1 & 1 & + & + & 3 \\
\hline Pinus sylvestris (c) & . & . & . & . & + & 1 & 1 & 1 \\
\hline Pleurozium shreberi & 2 & . & 4 & 3 & 5 & 5 & 5 & 4 \\
\hline Polytrichum juniperinum & . & . & . & 1 & 2 & 2 & 2 & 3 \\
\hline Pulsatilla patens & . & . & . & . & . & . & . & + \\
\hline Trientalis europaea & . & . & . & . & + & + & + & + \\
\hline Vaccinium uliginosum & . & . & . & . & . & + & + & 2 \\
\hline Vaccinium vitis-idaea & . & . & . & . & + & 1 & 1 & 1 \\
\hline \multicolumn{9}{|l|}{ Cl. Molinio-Arrhenatheretea } \\
\hline Achillea submillefolium & + & . & . & . & . & . & . & . \\
\hline Agrostis stolonifera & . & . & . & . & . & . & . & + \\
\hline
\end{tabular}




\begin{tabular}{|c|c|c|c|c|c|c|c|c|}
\hline \multirow{2}{*}{$\begin{array}{c}\text { Region } \\
\text { Relevé number }\end{array}$} & \multicolumn{3}{|c|}{ Right-bank Polesie } & \multicolumn{5}{|c|}{ Left-bank Polesie } \\
\hline & 1 & 2 & 3 & 4 & 5 & 6 & 7 & 8 \\
\hline Briza media & + & 1 & . & . & . & . & . & . \\
\hline Carex hirta & . & + & . & . & . & . & . & . \\
\hline Festuca rubra & . & . & 1 & 2 & . & . & . & . \\
\hline Holcus lanatus & . & 1 & . & . & . & . & . & . \\
\hline Ranunculus acris & . & + & . & . & . & . & . & . \\
\hline Sieglingia decumbens & . & . & 3 & . & . & . & . & . \\
\hline \multicolumn{9}{|l|}{ Cl. Nardetea strictae } \\
\hline Genista germanica & . & . & . & . & . & . & . & + \\
\hline Luzula campestris & . & . & . & . & . & + & + & + \\
\hline Lycopodium clavatum & . & . & + & . & + & + & + & . \\
\hline Nardus stricta & . & . & + & . & . & . & . & . \\
\hline Pilosella officinarum & + & + & + & + & + & + & . & + \\
\hline Potentilla erecta & . & + & . & . & + & . & . & + \\
\hline Veronica officinalis & + & . & . & + & + & + & + & . \\
\hline \multicolumn{9}{|l|}{ Cl. Koelerio-Corynephoretea canescentis } \\
\hline Chamaecytisus ruthenicus & . & . & + & . & . & . & . & . \\
\hline Festuca ovina & . & . & . & 2 & 3 & 3 & 3 & . \\
\hline Jasione montana & + & + & . & . & . & . & . & . \\
\hline Koeleria glauca & . & . & . & . & 2 & 3 & 3 & . \\
\hline Potentilla argentea & + & 1 & . & . & . & . & . & . \\
\hline Rumex acetosella & . & . & . & + & . & . & . & . \\
\hline Solidago virgaurea & . & . & + & . & . & . & . & . \\
\hline \multicolumn{9}{|l|}{ Cl. Crataego-Prunetea } \\
\hline Crataegus monogyna & + & + & . & . & . & . & . & . \\
\hline Galeopsis bifida & . & . & + & . & . & . & . & . \\
\hline Prunus spinosa & . & + & . & . & . & . & . & . \\
\hline Rosa canina & + & + & . & . & . & . & . & . \\
\hline \multicolumn{9}{|l|}{ Cl. Epilobietea angustifolii } \\
\hline Calamagrostis epigeios & + & 1 & . & + & . & . & . & . \\
\hline Rubus idaeus & . & . & . & . & + & . & . & . \\
\hline Sambucus racemosa & . & . & . & . & + & . & . & . \\
\hline \multicolumn{9}{|l|}{ Cl. Festuco-Brometea } \\
\hline Centaurea scabiosa & . & + & . & . & . & . & . & . \\
\hline Euphorbia cyparisias & + & 1 & . & . & . & . & . & . \\
\hline \multicolumn{9}{|l|}{ Cl. Trifolio-Geranietea sanguinei } \\
\hline Anemone sylvestris & + & · & . & . & $\cdot$ & . & . & . \\
\hline Galium verum & + & + & . & . & . & . & . & . \\
\hline
\end{tabular}




\begin{tabular}{|c|c|c|c|c|c|c|c|c|}
\hline \multirow{2}{*}{$\frac{\text { Region }}{\text { Relevé number }}$} & \multicolumn{3}{|c|}{ Right-bank Polesie } & \multicolumn{5}{|c|}{ Left-bank Polesie } \\
\hline & 1 & 2 & 3 & 4 & 5 & 6 & 7 & 8 \\
\hline Cl. Erico-Pinetea & & & & & & & & \\
\hline Daphne cneorum & + & . & . & . & . & . & . & . \\
\hline Cl. Papaveretea rhoeadis & & & & & & & & \\
\hline Viola arvensis & . & + & . & . & . & . & . & . \\
\hline Other species & & & & & & & & \\
\hline Betula pendula (b) & + & + & . & + & . & . & . & . \\
\hline Betula pendula (c) & . & + & . & . & . & . & . & . \\
\hline Calamagrostis arundinacea & . & . & . & . & . & . & . & 2 \\
\hline Cerasus mahaleb & + & . & . & . & . & . & . & . \\
\hline Genista tinctoria & . & . & + & . & . & . & . & . \\
\hline Holcus mollis & + & + & . & . & . & . & . & . \\
\hline Hypericum perforatum & + & + & . & 2 & . & . & . & . \\
\hline Populus tremula (b) & . & + & 1 & . & . & . & . & . \\
\hline Populus tremula (c) & . & + & + & $\cdot$ & . & . & . & . \\
\hline Pteridium aquilinum & $\cdot$ & . & . & + & . & . & . & . \\
\hline Pyrus communis (b) & + & 1 & . & . & . & . & . & . \\
\hline Quercus robur (b) & $\cdot$ & . & 1 & . & . & . & . & . \\
\hline Veronica chamaedrys & . & . & + & . & . & . & . & . \\
\hline
\end{tabular}

Notes. Dates, localities and authors of relevés:

1 - 12.08.2013, the Rivne shooting range, the Hoshchansk, Kostopil and Rivne districts, Rivne region; V. Melnyk.

2 - 21.07.2014, the shooting range near the northern outskirts of the Volodymyr-Volynskyi city, Volyn region; V. Melnyk.

3 - 21.08.1972, the Dubivka forestry, (sq. 36), Volodymyrets district, Rivne region; T. Andrienko.

$4-15.06 .2003$, the outkirts of the Lubenets village, Korop district, Chernihiv region; Yu. Karpenko.

5 - 10.06.2018, the Liubetskyi Masyv tract, Liubech forestry (sq. 20), Ripky district, Chernihiv region; O. Lukash, V. Popruha.

6 - 10.06.2018, the Liubetskyi Masyv tract, Liubech forestry (sq. 25), Ripky district, Chernihiv region; O. Lukash.

7 - 10.06.2018, the Liubetskyi Masyv tract, Liubech forestry (sq. 25), Ripky district, Chernihiv region; O. Lukash.

8 - 05.07.1980, the Chudivka forestry, (sq.30), Ripky district, Chernihiv region; T. Andrienko.

areas of 0.5-2 ha, and are distributed on sandy soils. Sometimes they represent a stage of a post-pyrogenic succession. The relevés in Table 2 give the idea of full composition and structure of the heathlands. Calluna vulgaris is a diagnostic species of the association. Its projective coverage varies from $15 \%$ to $90 \%$. Nardus stricta is a characteristic species of the Nardetea strictae class. Its role in phytocenoses is much smaller if compared with Calluna vulgaris. The qualitative and quantitative characteristics of these phytocenoses are different in Right-bank Polesie and Left-bank Polesie. Viola canina L. (the diagnostic species of the Calluno-Nardetum strictae association) in Right-bank Polesie, unlike in Left-bank Polesie, is not a constant component of phytocenoses. The characteristic species of the Violion caninae alliance are represented by Juncus squarrosus L. (in Right-bank Polesie) or Pimpinella saxifraga L. (in Leftbank Polesie). Right-bank heathlands are characterized by high constancy of Sieglingia decumbens (L.) Bernh, but it does not dominate. For comparison, this species dominates in communities of the Calluno-Nardetum strictae association in Poland (W. Matuszkiewicz, 2019), but it is absent in the relevés of Left-bank Polesie. The left-bank heathlands are characterized by high constancy of a number of characteristic species of the Molinio-Arrhenatheretea Tx. 1937 class and its syntaxons. For example, Gentiana pneumonanthe L., a regionally rare species of the Chernihiv region, is present in all the relevés. 


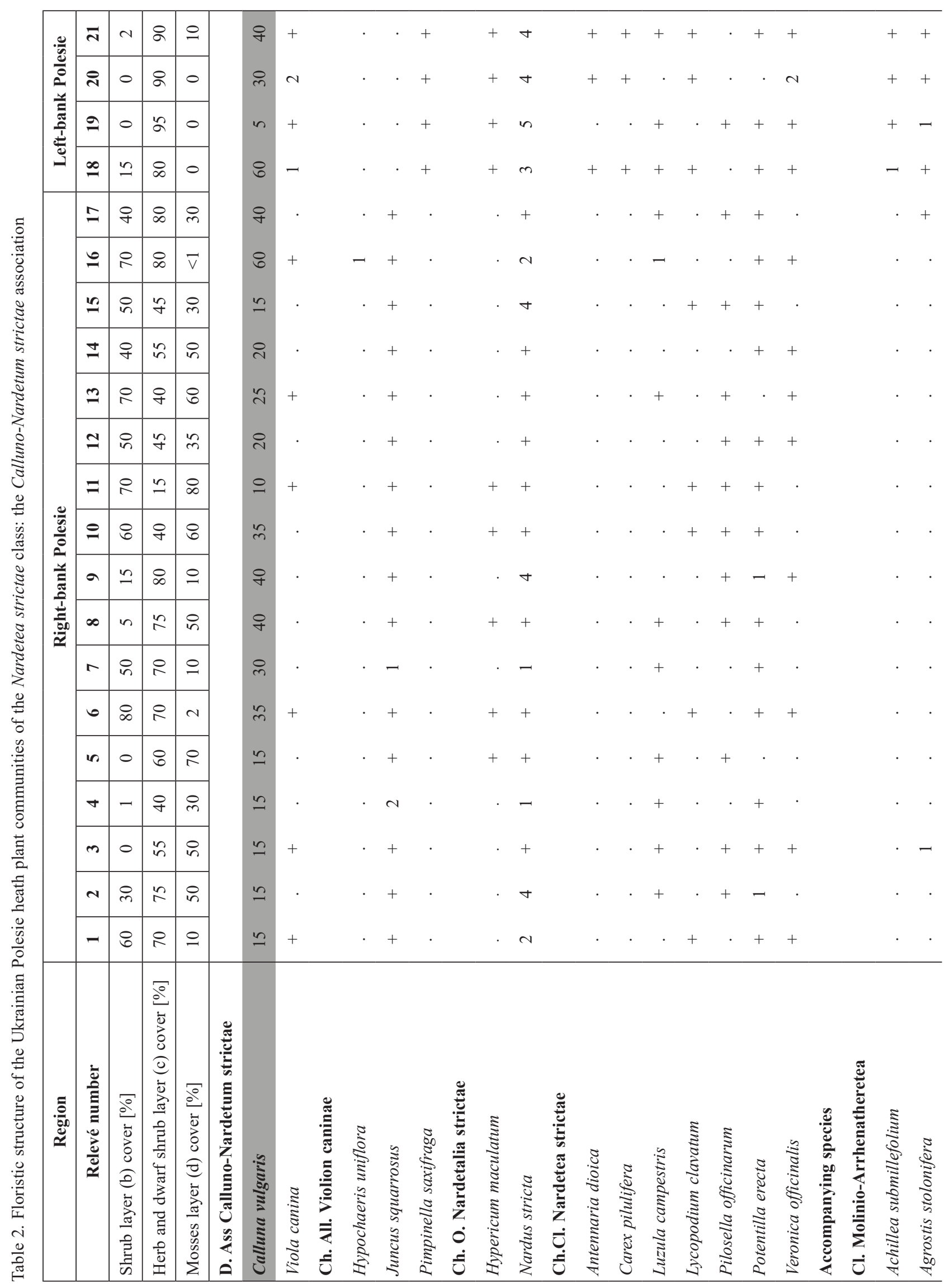




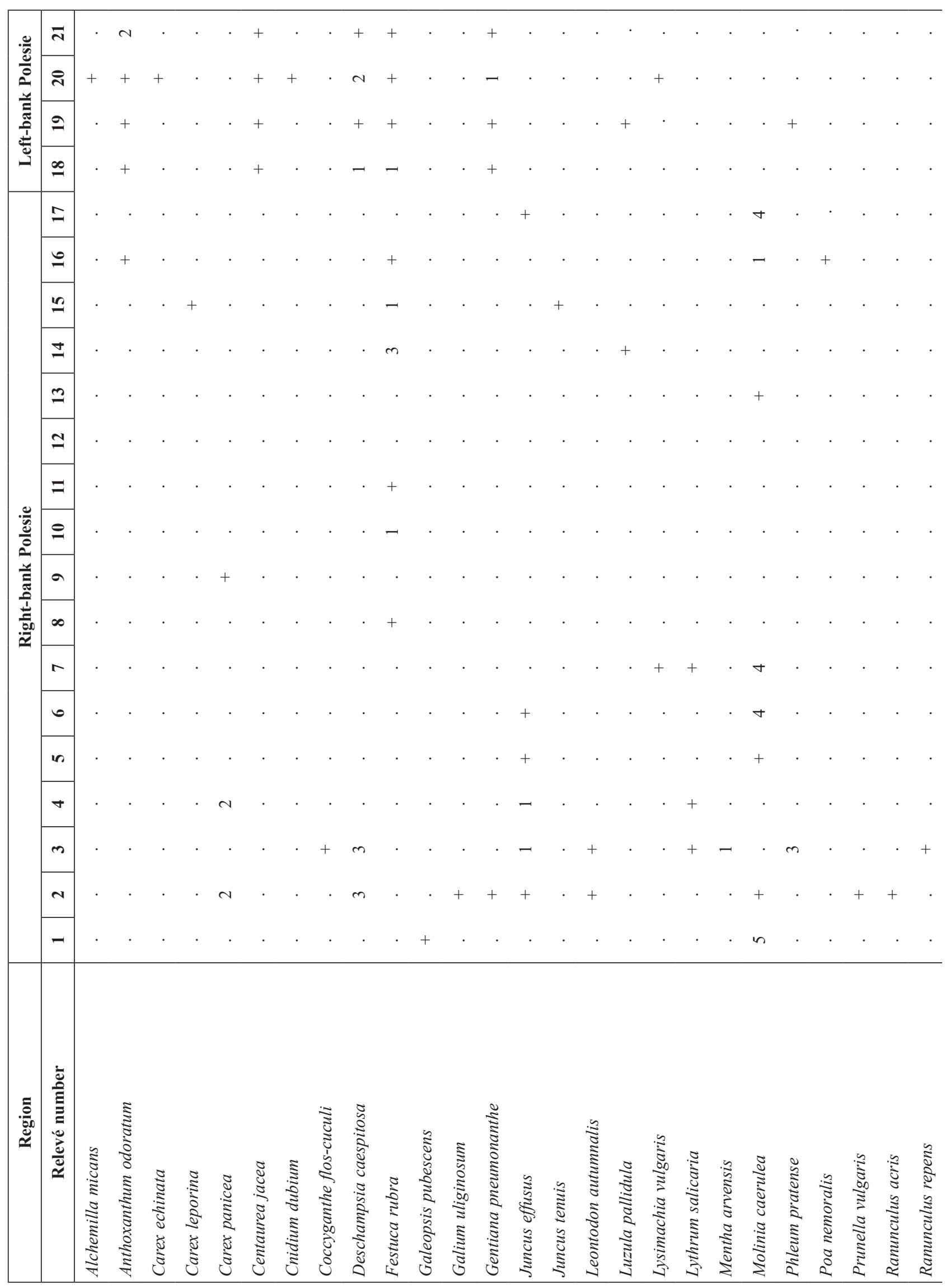




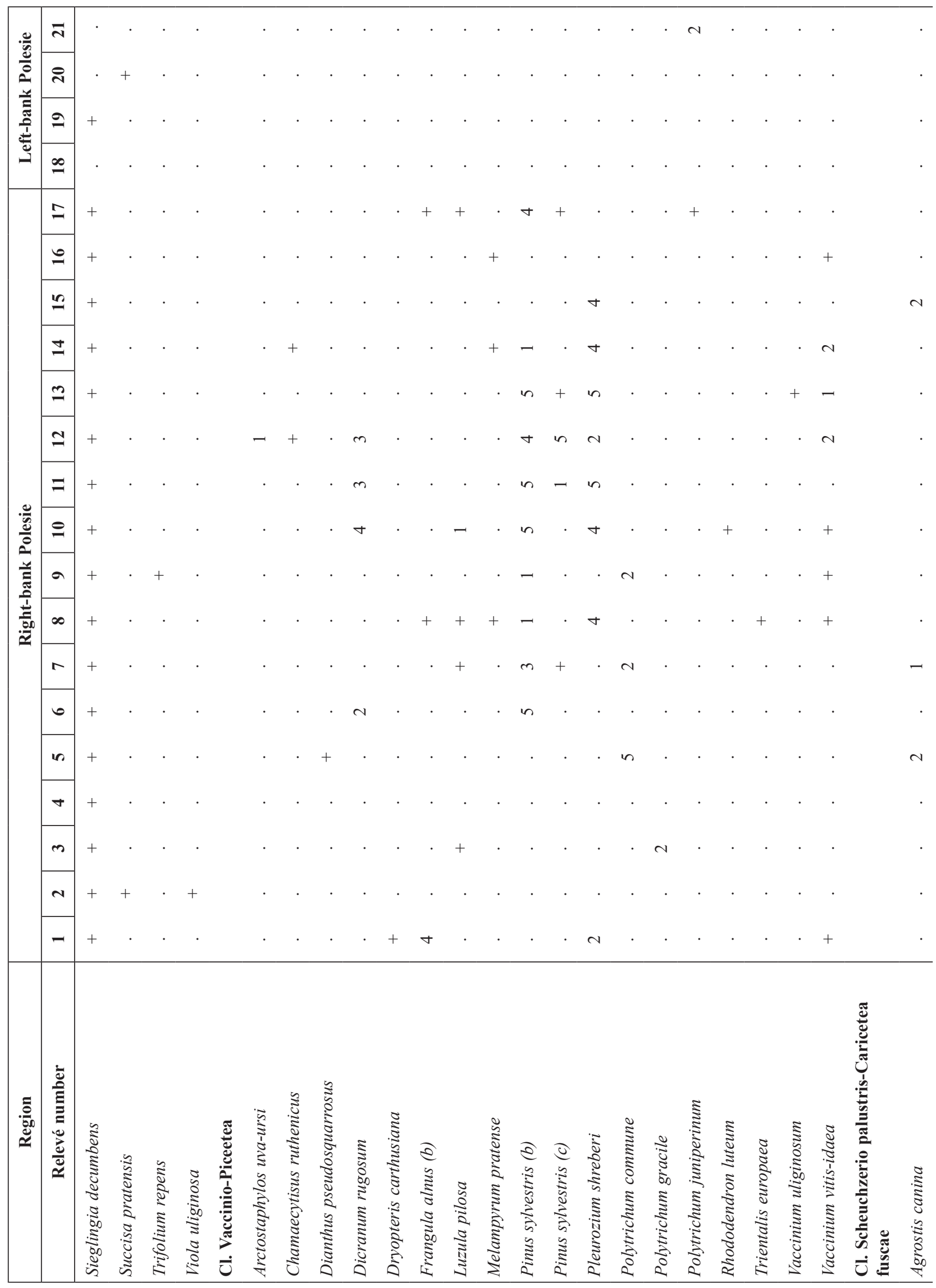




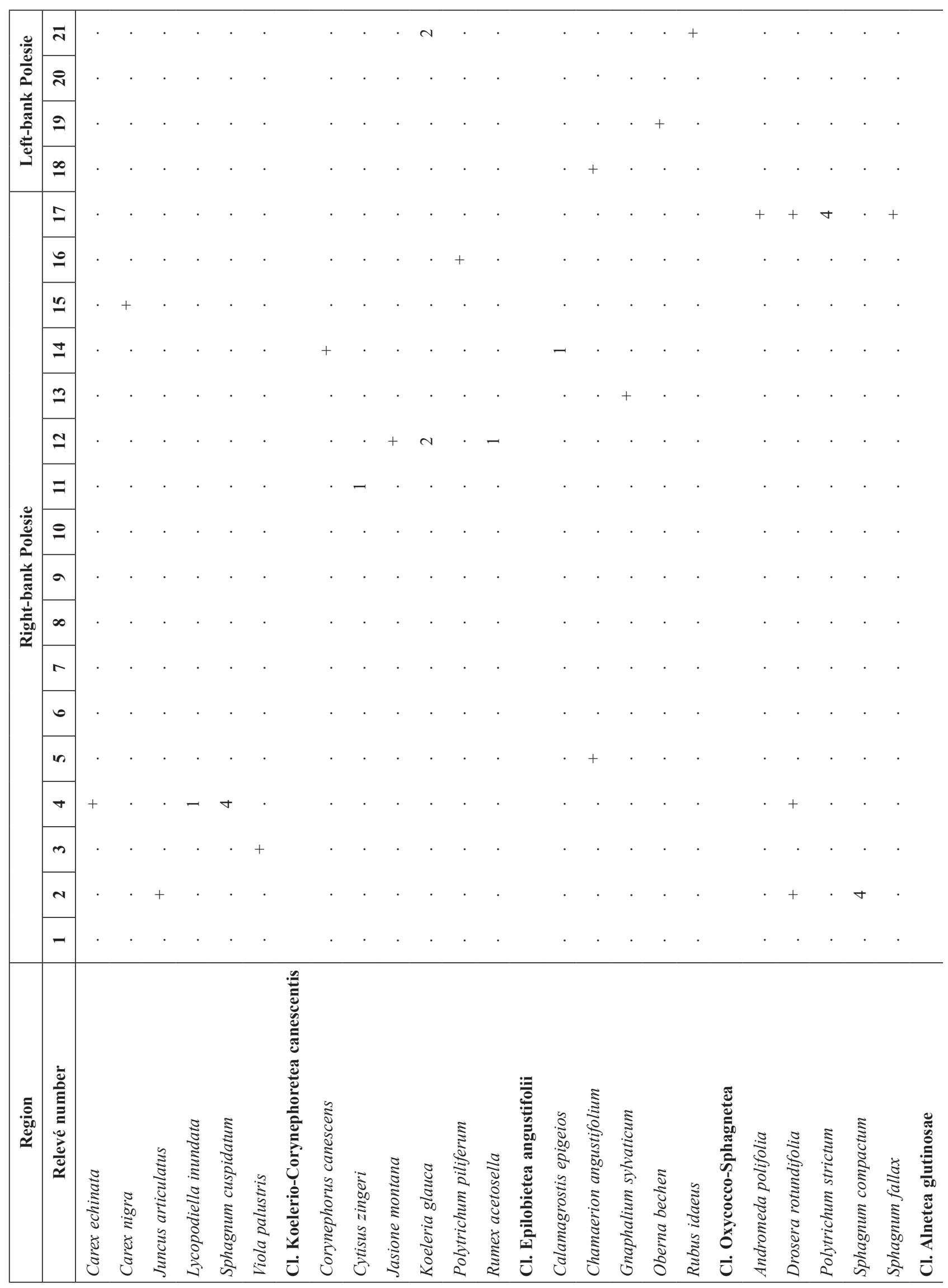




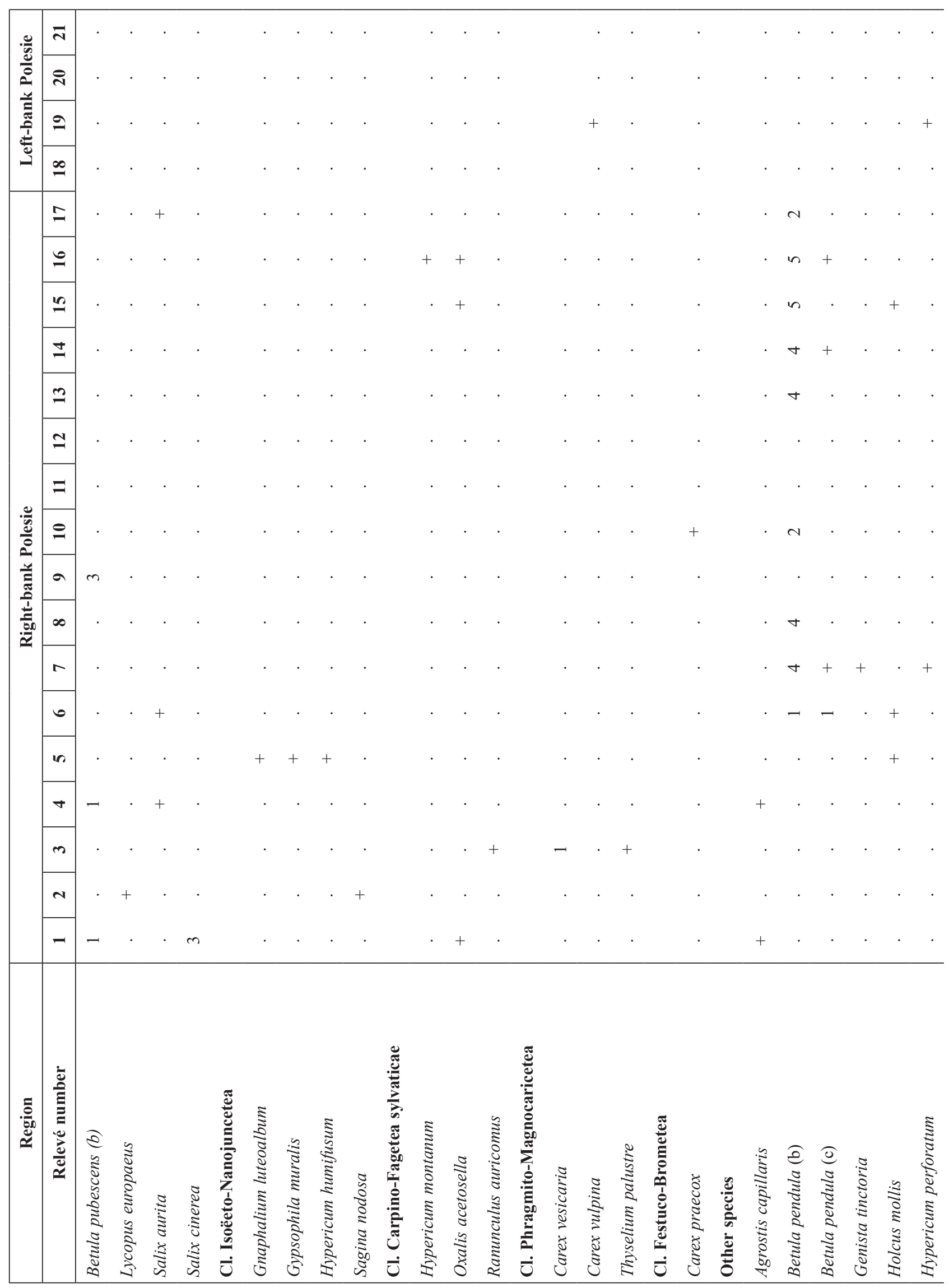



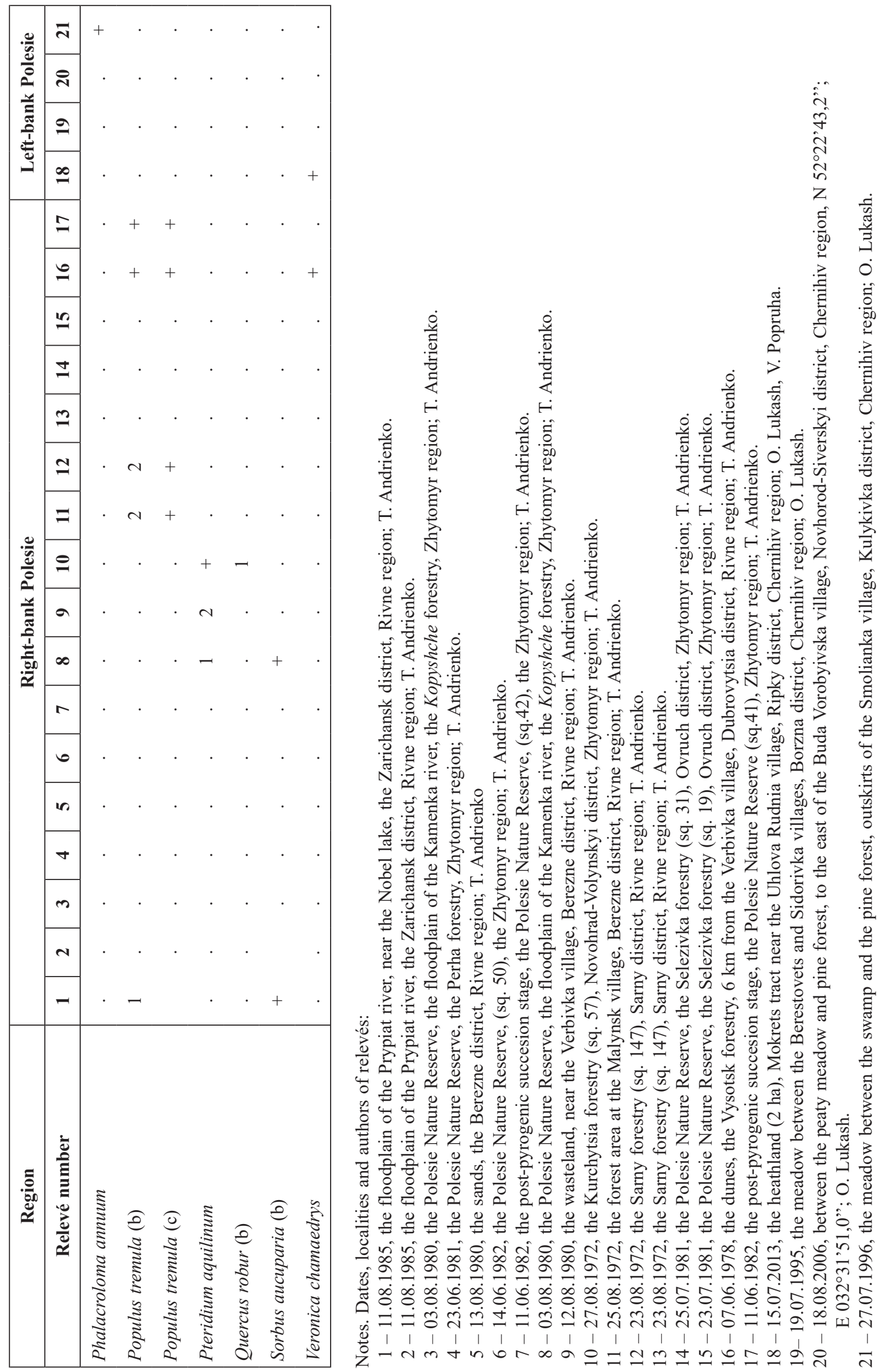
In Right-bank Polesie Calluna vulgaris is a component of phytocoenoses of dwarf-shrub, sedge and peatmoss vegetation of the Holarctic ombrotrophic bogs and wet heaths on extremely acidic soils. These phytocoenoses belong to the Oxycocco-Sphagnetea class (Table 3). There are no such biotopes in Left-bank Polesie, as they were destroyed in 1970s-1980s as a result of drainage reclamation. Most of plant communities of the Oxycocco-Sphagnetea class, represented by the Sphagnetalia medii order and the Sphagnion medii alliance, can be seen in the Polesie Nature
Reserve (the Zhytomyr region). Calluna vulgaris participation in mire phytocenoses varies depending on a stage of mire development. The highest projective coverage of Calluna vulgaris (up to 60-80\%) is observed in succession stages after wildfires. All the forest oligotrophic bogs with Calluna vulgaris (Table 3), described by prof. T. Andrienko, were identified, according to W. Matuszkiewicz (2019), as belonging to the Ledo-Sphagnetum magellanici association, because Ledum palustre L. (the diagnostic species of this association) was present in all relevés.

Table 3. Floristic structure of the forest bogs plant communities (Ledo-Sphagnetum magellanici association) with Calluna vulgaris

\begin{tabular}{|c|c|c|c|c|c|c|c|c|c|c|c|c|c|c|c|c|c|}
\hline Relevé number & 1 & 2 & 3 & 4 & 5 & 6 & 7 & 8 & 9 & 10 & 11 & 12 & 13 & 14 & 15 & 16 & 17 \\
\hline Tree layer (a) cover $[\%]$ & 0 & 5 & 0 & 0 & 0 & 50 & 0 & 0 & 40 & 40 & 0 & 0 & 70 & 0 & 40 & 0 & 0 \\
\hline Shrub layer (b) cover [\%] & 50 & 50 & 50 & 60 & 30 & 10 & 40 & 40 & 20 & 75 & 70 & 40 & 20 & 40 & $<1$ & 20 & 50 \\
\hline $\begin{array}{l}\text { Herb and dwarf shrub layer (c) } \\
\text { cover }[\%]\end{array}$ & 60 & 75 & 80 & 80 & 65 & 60 & 80 & 60 & 60 & 75 & 70 & 70 & 80 & 60 & 65 & 80 & 90 \\
\hline Mosses layer (d) cover [\%] & 70 & 20 & 20 & 40 & 80 & 60 & 95 & 98 & 90 & 80 & 40 & 70 & 40 & 95 & 98 & 30 & 0 \\
\hline \multicolumn{18}{|l|}{$\begin{array}{l}\text { D. Ass. Ledo-Sphagnetum } \\
\text { magellanici }\end{array}$} \\
\hline Ledum palustre & 1 & + & + & + & + & 4 & 1 & + & 3 & 1 & + & + & 4 & 1 & 1 & 1 & 1 \\
\hline Oxycoccus microcarpus & . & . & . & . & . & . & . & + & . & + & . & $\cdot$ & . & + & . & . & \\
\hline \multicolumn{18}{|l|}{$\begin{array}{l}\text { D. Ass. Gr. of the forest bogs } \\
\text { (Ch. Cl. Vaccinio-Piceetea) }\end{array}$} \\
\hline Pinus sylvestris (a) & . & 1 & . & . & . & 4 & . & . & 4 & 1 & . & . & 5 & . & 4 & . & . \\
\hline Pinus sylvestris (b) & 3 & 2 & 1 & 1 & 3 & 1 & 4 & 4 & 3 & 4 & 5 & 4 & + & 4 & + & + & + \\
\hline Pinus sylvestris (c) & + & . & . & . & . & . & . & . & . & . & & + & + & 1 & . & . & . \\
\hline Pleurozium shreberi & + & . & . & . & . & . & + & . & . & . & 3 & . & 4 & . & . & . & 1 \\
\hline Trientalis europaea & . & . & . & . & + & . & . & . & + & . & . & . & . & . & . & + & . \\
\hline Vaccinium myrtillus & . & + & . & . & . & + & + & . & . & . & . & . & . & . & . & . & + \\
\hline Vaccinium uliginosum & + & 1 & + & . & . & + & + & . & . & + & + & . & + & . & . & . & 2 \\
\hline Vaccinium vitis-idaea & 1 & + & . & . & . & . & . & . & . & . & + & . & 1 & . & . & + & . \\
\hline \multicolumn{18}{|l|}{$\begin{array}{l}\text { Ch. All. Sphagnion medii } \\
\text { and O. Sphagnetalia medii }\end{array}$} \\
\hline Andromeda polifolia & . & . & + & + & 1 & + & + & + & 1 & + & + & . & + & 1 & 2 & + & . \\
\hline Eriophorum vaginatum & . & . & . & + & 4 & 1 & 4 & 4 & 3 & 1 & 1 & + & 1 & 4 & 4 & 4 & + \\
\hline Oxycoccus palustris & . & . & 1 & . & 1 & + & 3 & + & 2 & 3 & . & . & + & 1 & 1 & 1 & . \\
\hline Sphagnum acutifolium & 4 & . & . & . & . & 3 & 3 & . & . & . & 3 & . & . & . & . & . & . \\
\hline Sphagnum centrale & . & . & . & . & . & . & . & . & . & . & . & . & + & . & 5 & + & . \\
\hline Sphagnum compactum & . & . & . & . & . & . & . & . & . & . & . & . & . & . & . & . & 3 \\
\hline
\end{tabular}




\begin{tabular}{|c|c|c|c|c|c|c|c|c|c|c|c|c|c|c|c|c|c|}
\hline Relevé number & 1 & 2 & 3 & 4 & 5 & 6 & 7 & 8 & 9 & 10 & 11 & 12 & 13 & 14 & 15 & 16 & 17 \\
\hline Sphagnum cuspidatum & . & . & 1 & 4 & 2 & & . & 5 & 5 & . & . & 4 & . & . & . & $\cdot$ & . \\
\hline Sphagnum fallax & . & . & . & . & 4 & . & 5 & . & . & . & . & . & . & . & . & . & . \\
\hline Sphagnum fuscum & . & 3 & . & . & . & . & . & . & . & . & . & . & + & . & 1 & . & . \\
\hline Sphagnum magellanicum & + & + & + & . & 3 & . & . & 3 & 2 & . & + & . & . & + & . & 4 & + \\
\hline Sphagnum rubellum & . & . & . & . & . & . & . & . & . & 5 & . & + & . & 5 & 1 & + & . \\
\hline \multicolumn{18}{|c|}{ Ch. Cl. Oxycocco-Sphagnetea } \\
\hline Aulacomnium palustre & . & . & + & . & . & 3 & + & + & . & . & . & 2 & . & . & . & . & + \\
\hline Drosera rotundifolia & + & + & + & + & + & . & . & + & + & + & + & . & + & + & + & + & . \\
\hline Salix aurita & . & + & . & + & + & . & . & . & . & . & . & . & . & . & . & . & . \\
\hline \multicolumn{18}{|l|}{ Accompanying species } \\
\hline \multicolumn{18}{|l|}{ Cl. Alnetea glutinosae } \\
\hline Alnus glutinosa (b) & + & . & . & . & . & . & . & . & . & . & . & . & . & . & . & . & . \\
\hline Betula pubescens (a) & . & . & . & . & . & 2 & . & . & . & . & . & . & . & . & . & . & . \\
\hline Betula pubescens (b) & . & . & 4 & 5 & . & 2 & . & . & . & 2 & 1 & 2 & . & . & . & 3 & 4 \\
\hline Betula pubescens (c) & . & . & . & . & . & . & . & . & . & . & . & + & . & . & . & . & . \\
\hline Salix cinerea & + & + & . & . & . & . & . & . & . & . & . & . & . & . & . & . & . \\
\hline \multicolumn{18}{|l|}{ Cl. Vaccinio-Piceetea } \\
\hline Dicranum rugosum & . & . & . & . & . & . & . & . & . & . & . & . & 1 & . & . & . & . \\
\hline Melampyrum pratense & + & . & . & . & . & . & . & . & . & . & . & . & . & . & . & . & . \\
\hline Polytrichum commune & + & . & . & 1 & . & . & . & . & . & . & . & 4 & . & . & . & . & $\cdot$ \\
\hline Polytrichum gracile & . & 1 & . & . & . & . & . & . & . & . & . & . & . & . & . & . & . \\
\hline \multicolumn{18}{|l|}{$\begin{array}{l}\text { Cl. Scheuchzerio palustris- } \\
\text { Caricetea fuscae }\end{array}$} \\
\hline Carex lasiocarpa & . & . & . & 2 & 1 & . & . & . & . & + & . & . & . & + & . & . & . \\
\hline Carex nigra & + & + & . & . & . & . & . & . & . & . & . & + & . & . & . & . & . \\
\hline Rhynchospora alba & . & + & . & 1 & . & . & . & . & . & . & . & . & . & . & . & + & . \\
\hline \multicolumn{18}{|c|}{ Cl. Molinio-Arrhenatheretea } \\
\hline Molinia caerulea & 2 & 2 & . & . & . & . & + & . & . & + & 2 & 4 & 3 & . & . & . & . \\
\hline Juncus effusus & 1 & . & . & . & . & . & . & . & . & . & . & + & . & . & . & . & . \\
\hline \multicolumn{18}{|l|}{ Cl. Nardetea strictae } \\
\hline Nardus stricta & . & + & . & . & . & . & . & . & . & . & . & . & . & . & . & . & . \\
\hline Potentilla erecta & . & + & . & . & . & . & . & . & . & . & . & + & . & . & . & . & . \\
\hline \multicolumn{18}{|l|}{ Cl. Calluno-Ulicetea } \\
\hline Arctostaphylos uva-ursi & $\cdot$ & . & . & . & . & . & . & . & + & . & . & . & . & . & . & . & . \\
\hline Calluna vulgaris & 40 & 60 & 65 & 60 & 1 & 5 & 2 & 1 & 10 & 50 & 60 & 25 & 1 & 5 & 10 & 30 & 80 \\
\hline
\end{tabular}




\begin{tabular}{|c|c|c|c|c|c|c|c|c|c|c|c|c|c|c|c|c|c|}
\hline Relevé number & 1 & 2 & 3 & 4 & 5 & 6 & 7 & 8 & 9 & 10 & 11 & 12 & 13 & 14 & 15 & 16 & 17 \\
\hline \multicolumn{18}{|c|}{$\begin{array}{l}\text { Cl. Koelerio-Corynephoretea } \\
\text { canescentis }\end{array}$} \\
\hline Astragalus arenarius & . & . & . & . & . & . & . & . & + & . & . & . & . & . & . & . & . \\
\hline \multicolumn{18}{|l|}{ Other species } \\
\hline Betula pendula (a) & . & . & . & . & . & . & + & . & . & . & . & . & . & . & 3 & . & . \\
\hline Betula pendula (b) & 4 & 4 & . & . & 5 & . & . & . & . & . & . & . & 3 & 2 & + & . & . \\
\hline Betula pendula (c) & . & 2 & . & . & . & . & . & . & . & . & . & . & 2 & + & + & . & . \\
\hline Populus tremula (b) & . & 1 & + & + & . & . & . & . & . & . & . & . & . & . & . & + & . \\
\hline Populus tremula (c) & . & . & . & . & . & . & . & . & . & . & . & . & . & . & . & + & . \\
\hline Pteridium aquilinum & . & + & . & . & . & . & . & . & . & . & . & . & . & . & . & . & . \\
\hline Polytrichum alpestre & . & . & 3 & . & . & 4 & + & 1 & 2 & . & . & . & . & . & . & . & . \\
\hline
\end{tabular}

Notes.

Dates and localities of relevés:

$1-16.08 .1980$, the Sarny forestry (sq. 9), Sarny district, Rivne region.

$2-13.08 .1980$, the post-pyrogenic succession stage, the Berezne district, Rivne region.

$3-10.08 .1980$, the post-pyrogenic succession stage, the Pochaiv Reserve (sq. 76), Berezne district, Rivne region.

$4-10.08 .1980$, the post-pyrogenic succession stage, the Pochaiv Reserve (sq. 76), Berezne district, Rivne region.

$5-12.07 .1982$, the Plotnytsia tract, Chervona Volia forestry (sq. 7), Novohrad-Volynskyi district, Zhytomyr region.

6 - 10.07.1982, the Chervona Volia forestry (sq. 1), Novohrad-Volynskyi district, Zhytomyr region.

7 - 22.10.1974, the Perebrody forestry (sq. 81), Dubrovytsia district, Rivne region.

8 - 27.07.1976, the Rostan forestry (sq. 21), Shatsk district, Volyn region.

9 - 16.07.1973, the Babii Mokh swamp, Zolote forestry (sq. 39), Dubrovytsia district, Rivne region.

10 - 25.06.1981, the Polesie Nature Reserve, the Klitne tract, Kopyshche forestry (sq. 47), Zhytomyr region.

$11-25.06 .1981$, the post-pyrogenic succession stage, the Polesie Nature Reserve, the Klitne tract, Kopyshche forestry (sq. 38), Zhytomyr region.

12 - 25.06.1981, the Polesie Nature Reserve, Kopyshche forestry (sq. 26), Zhytomyr region.

13 - 13.06.1982, the swamp forest, the Polesie Nature Reserve (sq. 51), Zhytomyr region.

14 - 12.06.1982, the oligotrophic swamp, the Polesie Nature Reserve (sq. 40), Zhytomyr region.

15 - 12.06.1982, the oligotrophic swamp, the Polesie Nature Reserve (sq. 41), Zhytomyr region.

$16-11.06 .1982$, the post-pyrogenic succession stage, the Polesie Nature Reserve (sq. 41), Zhytomyr region.

$17-07.06 .1978$, the Vysotsk forestry (sq. 75), near the Verbivka village, Dubrovytsia district, Rivne region.

Author of relevés: T. Andrienko.

The European temperate and subboreal pine forests on nutrient-poor acidic sandy soils (the Vaccinio-Piceetea class) are typical and most common habitats of Calluna vulgaris in Ukrainian Polesie. The species occurs in phytocenoses mainly individually. The maximum projective coverage of Calluna vulgaris was observed in pine, birch-pine and oak-pine forests of 30-40 years of age. Tables 4 and 5 provide information on structure and floristic composition of forest phytocenoses with Calluna vulgaris, which projective coverage is over $1 \%$. As it can be seen, all these plant communities were well diagnosed by characteristic and differentiating species. The relationships between the values of tree layer and projective coverage of Calluna vulgaris were not found.
The maximum projective coverage of Calluna vulgaris in plant communities of the Pinetalia sylvestris order associations (the Dicrano-Pinion sylvestris alliance) in Rightbank Polesie respectively is: Querco-Piceetum $-45 \%$, Cladonio-Pinetum - 40\%, Molinio-Pinetum - 35\%, Peucedano-Pinetum - 30\%, Vaccinio uliginosi-Pinetum - 10\% (Table 4, relevés 1-26). Calluna vulgaris coverage of $40 \%$ was observed only in the phytocenoses of the Cladonio-Pinetum association (Table 5, relevés 1-5) in Left-bank Polesie. The projective coverage of Calluna vulgaris in other plant communities of pine and mixed forests of Left-bank Polesie does not exceed 5\% (Table 5, relevés 6-23). 


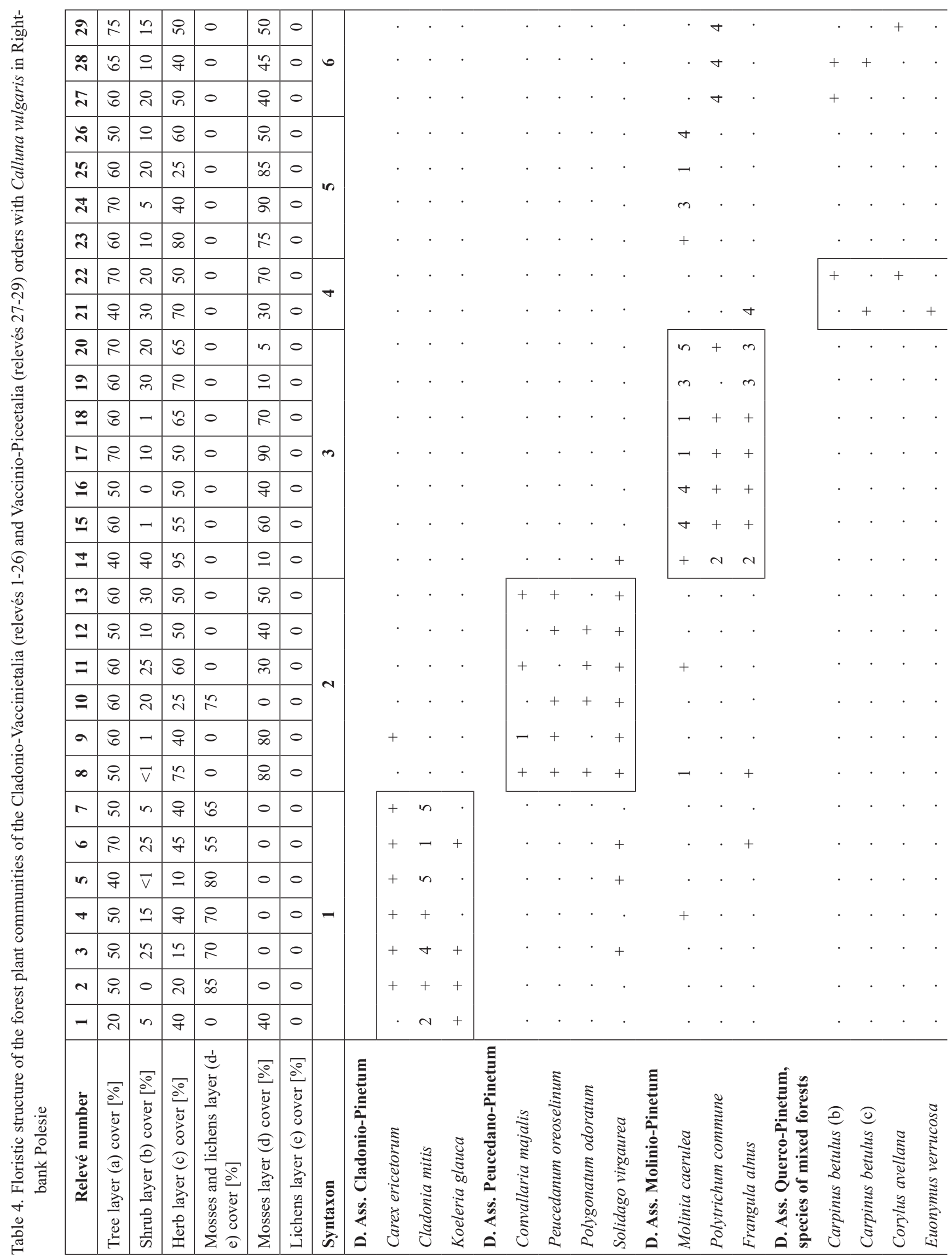




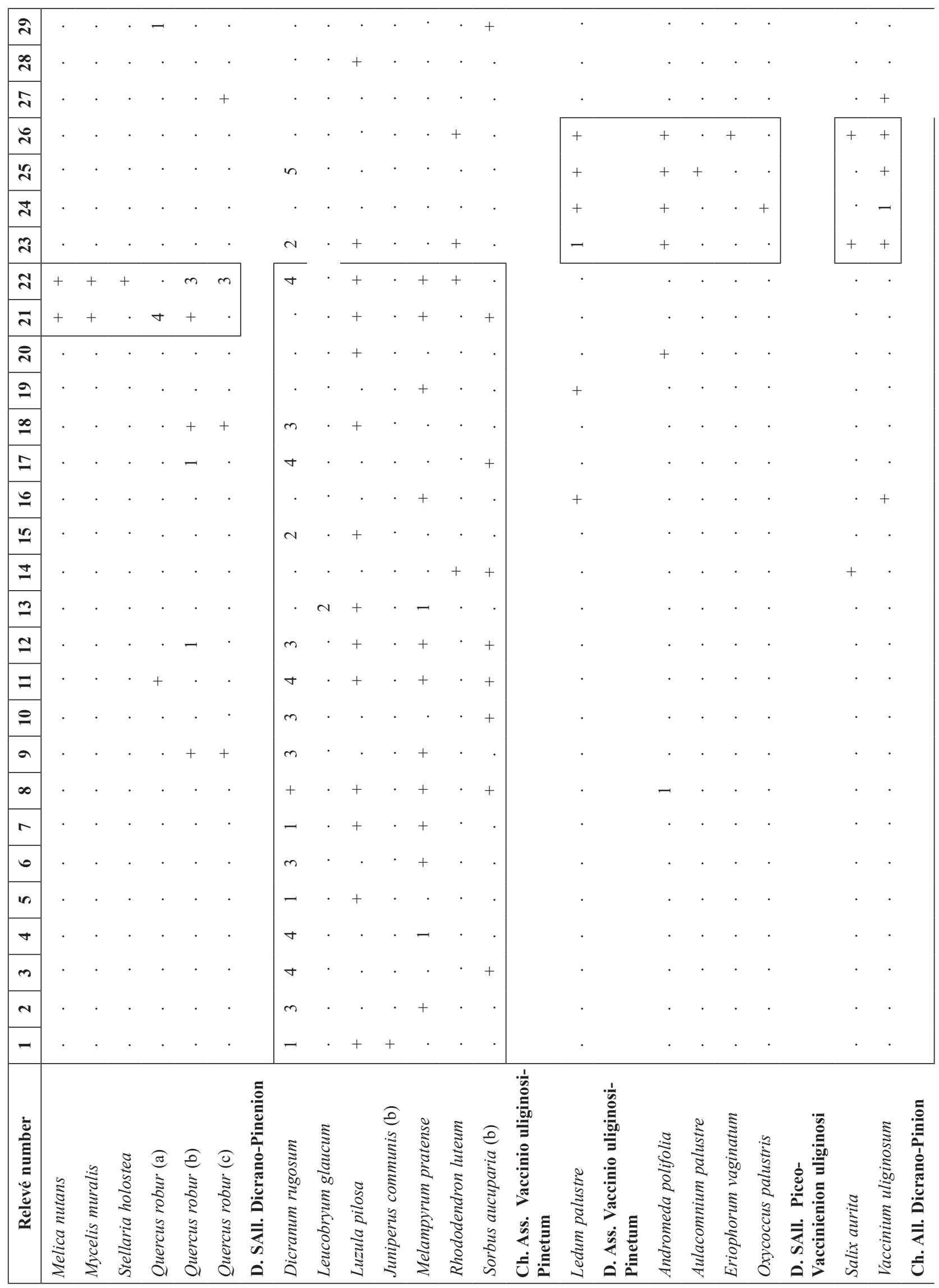




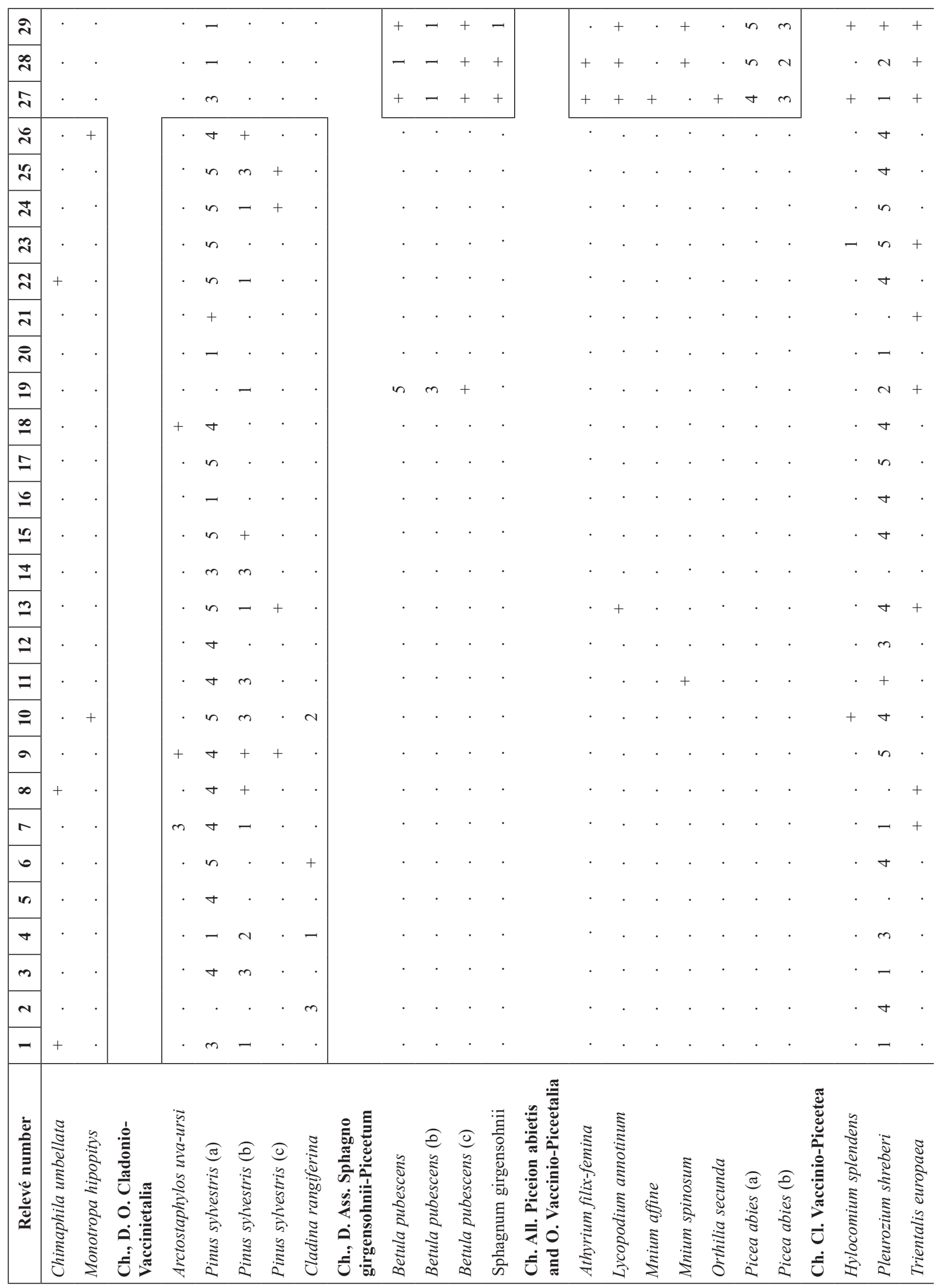




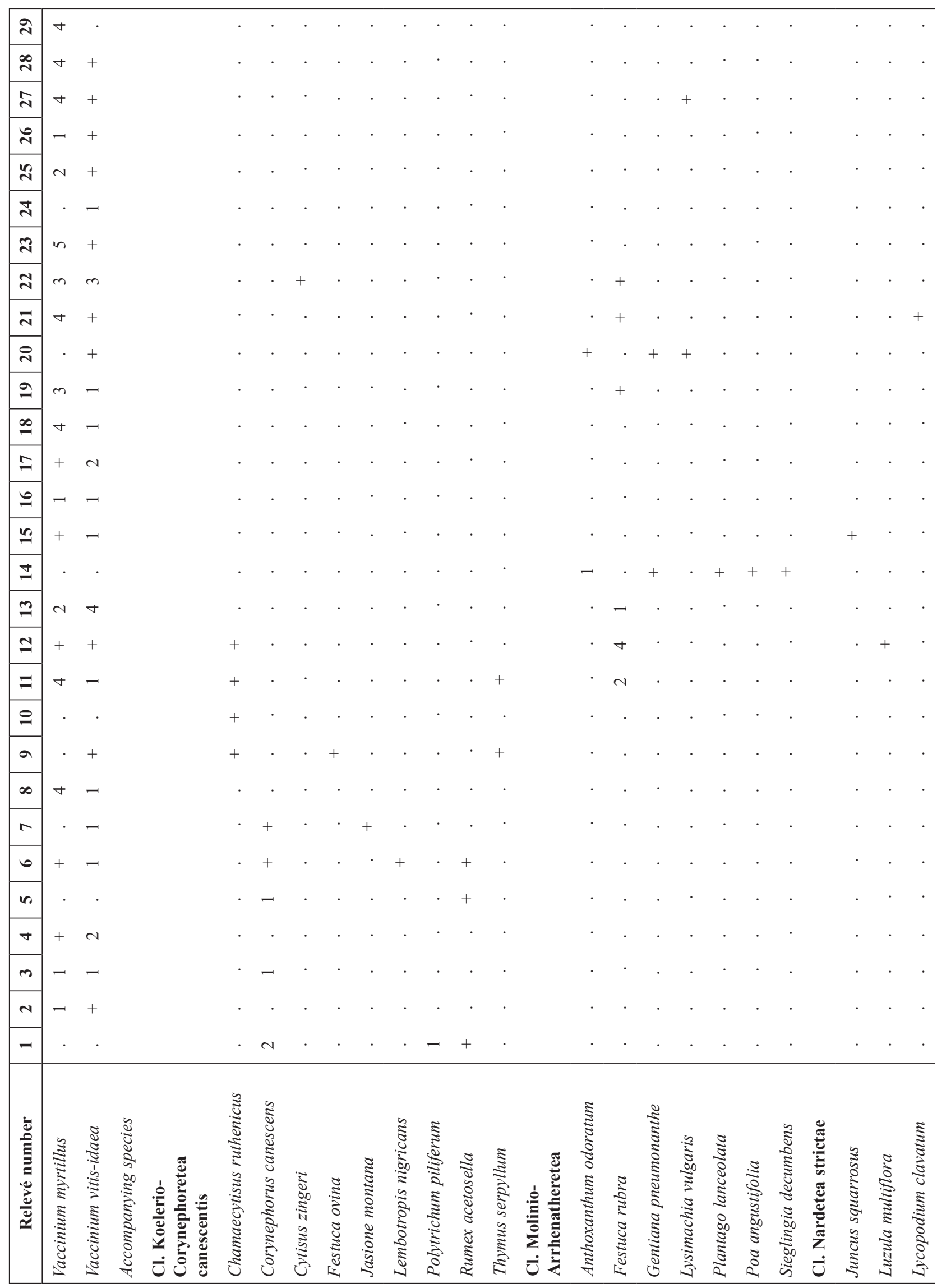




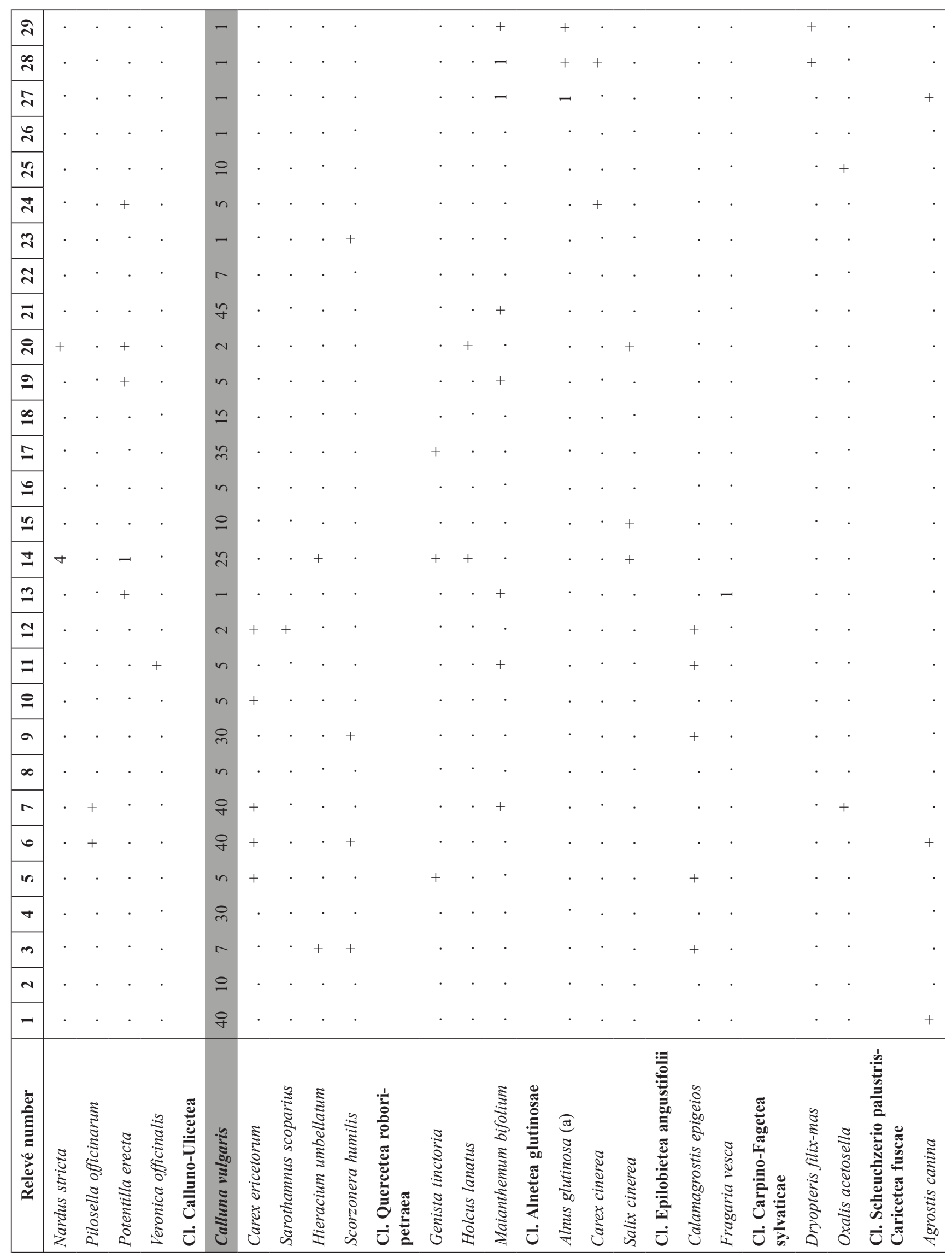




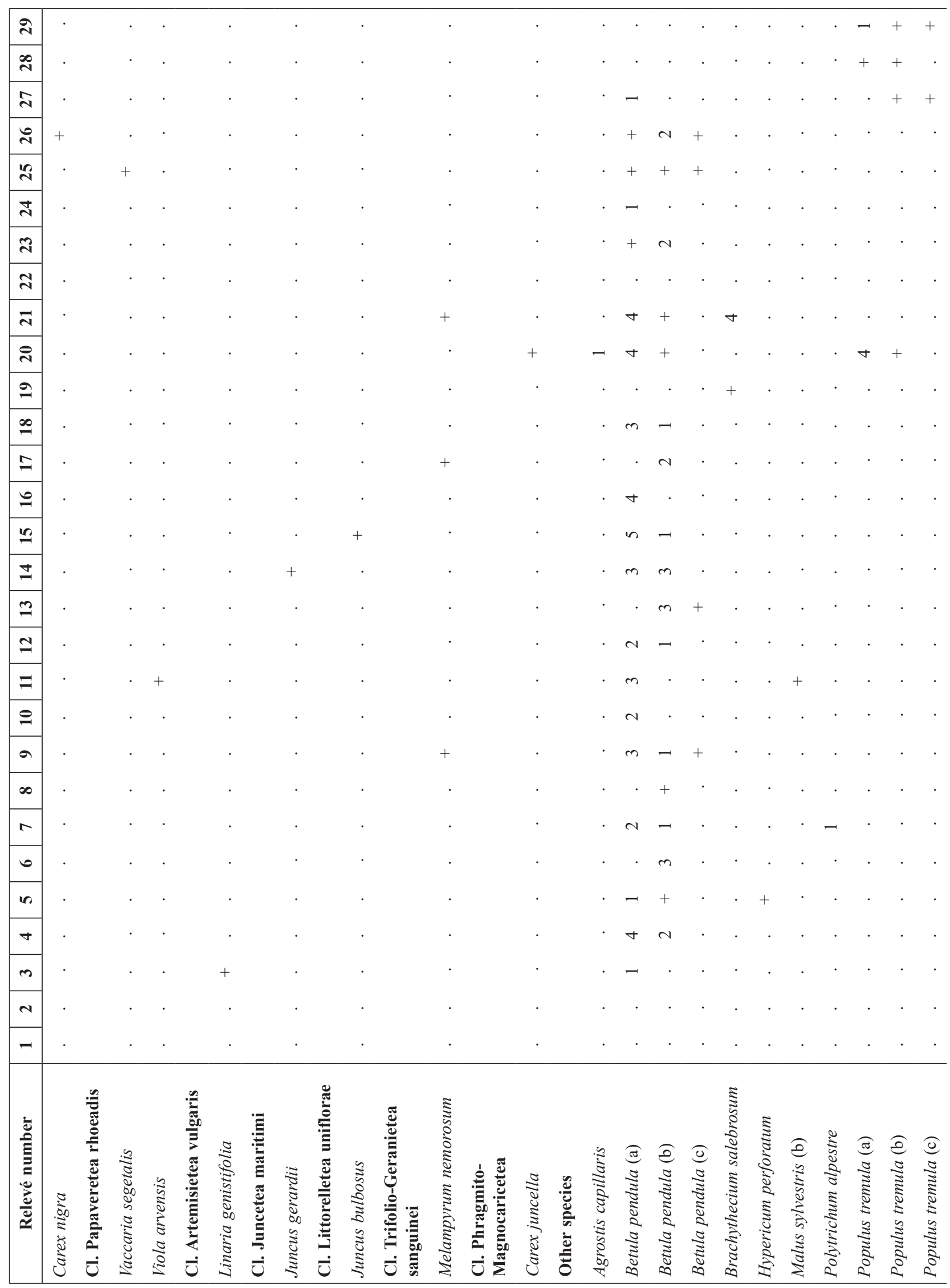




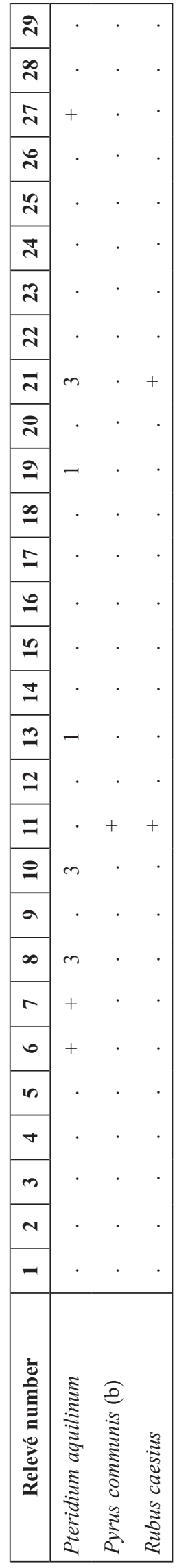

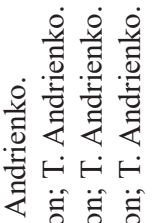

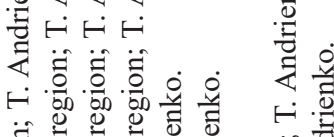

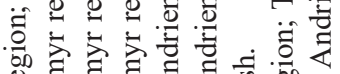

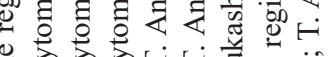

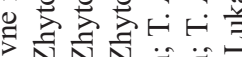

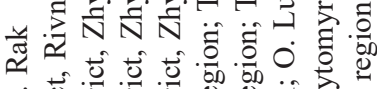

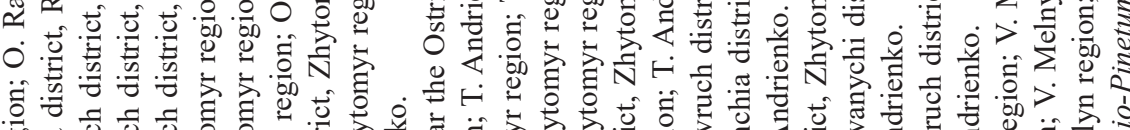

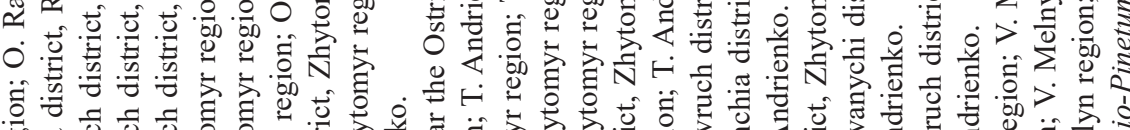

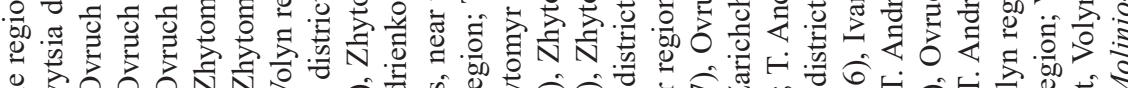

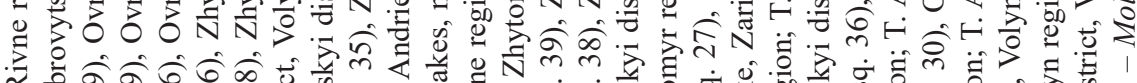
«

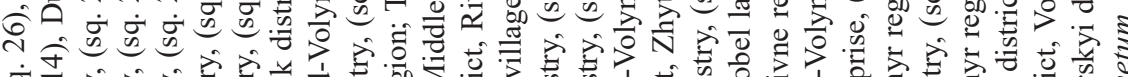

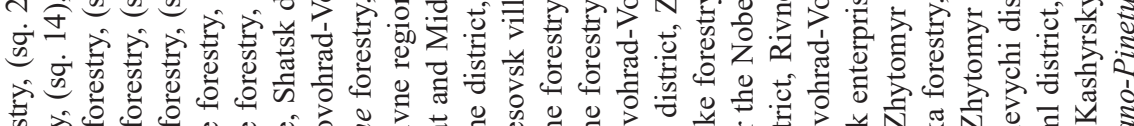

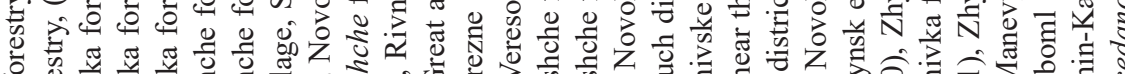

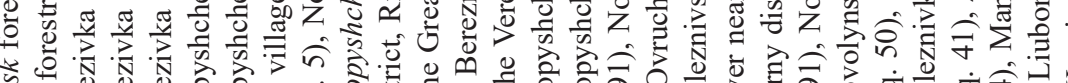

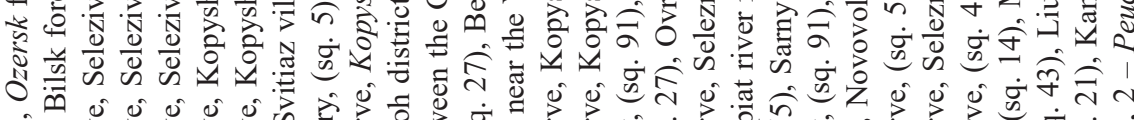

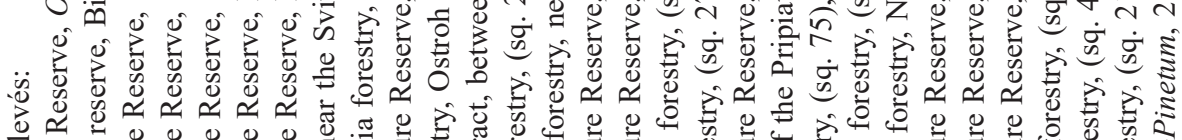

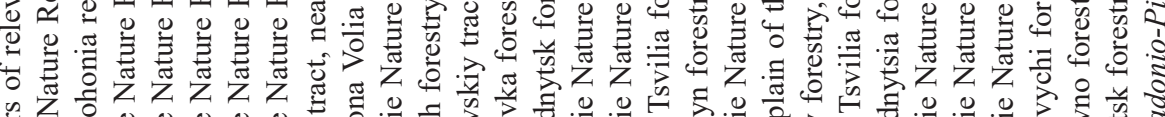

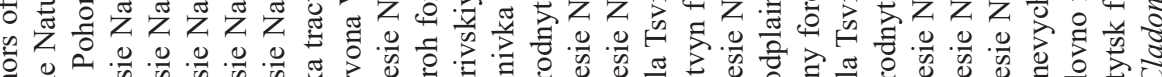

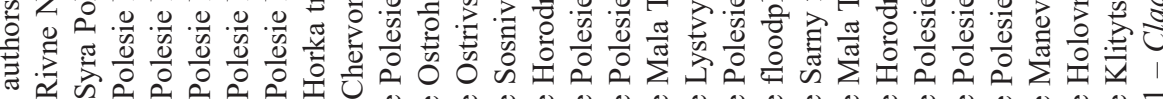

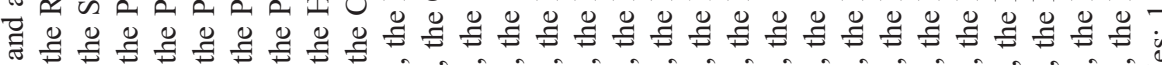

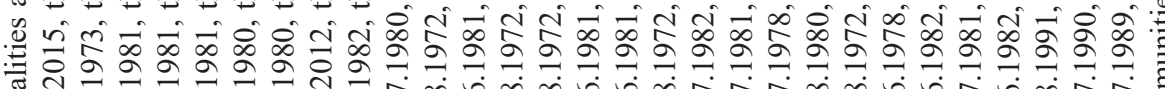

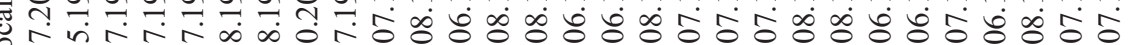
ब.

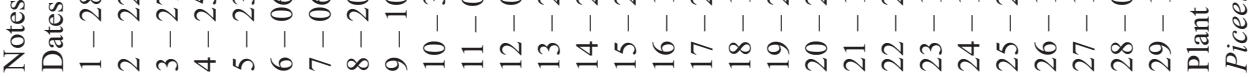




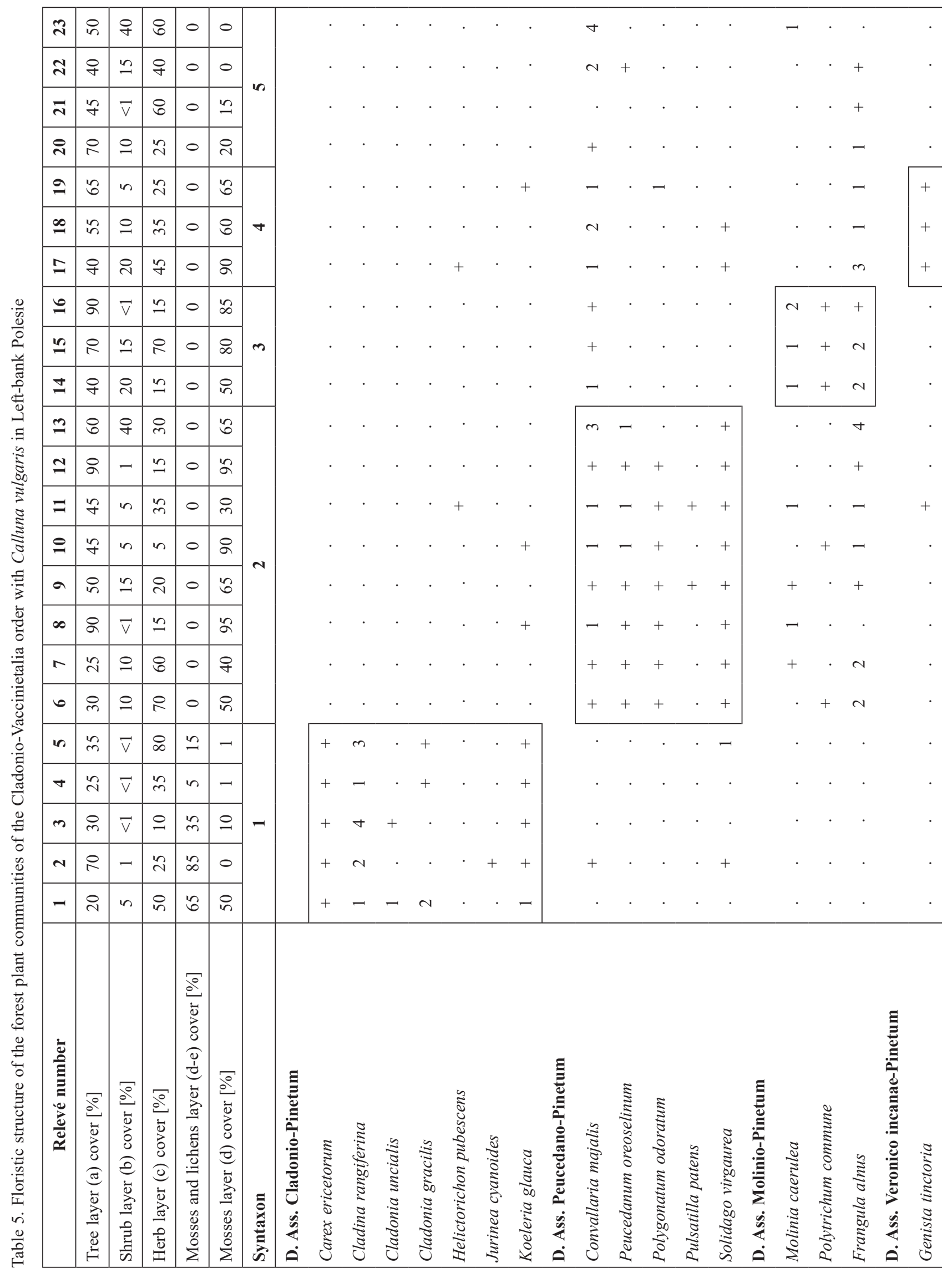




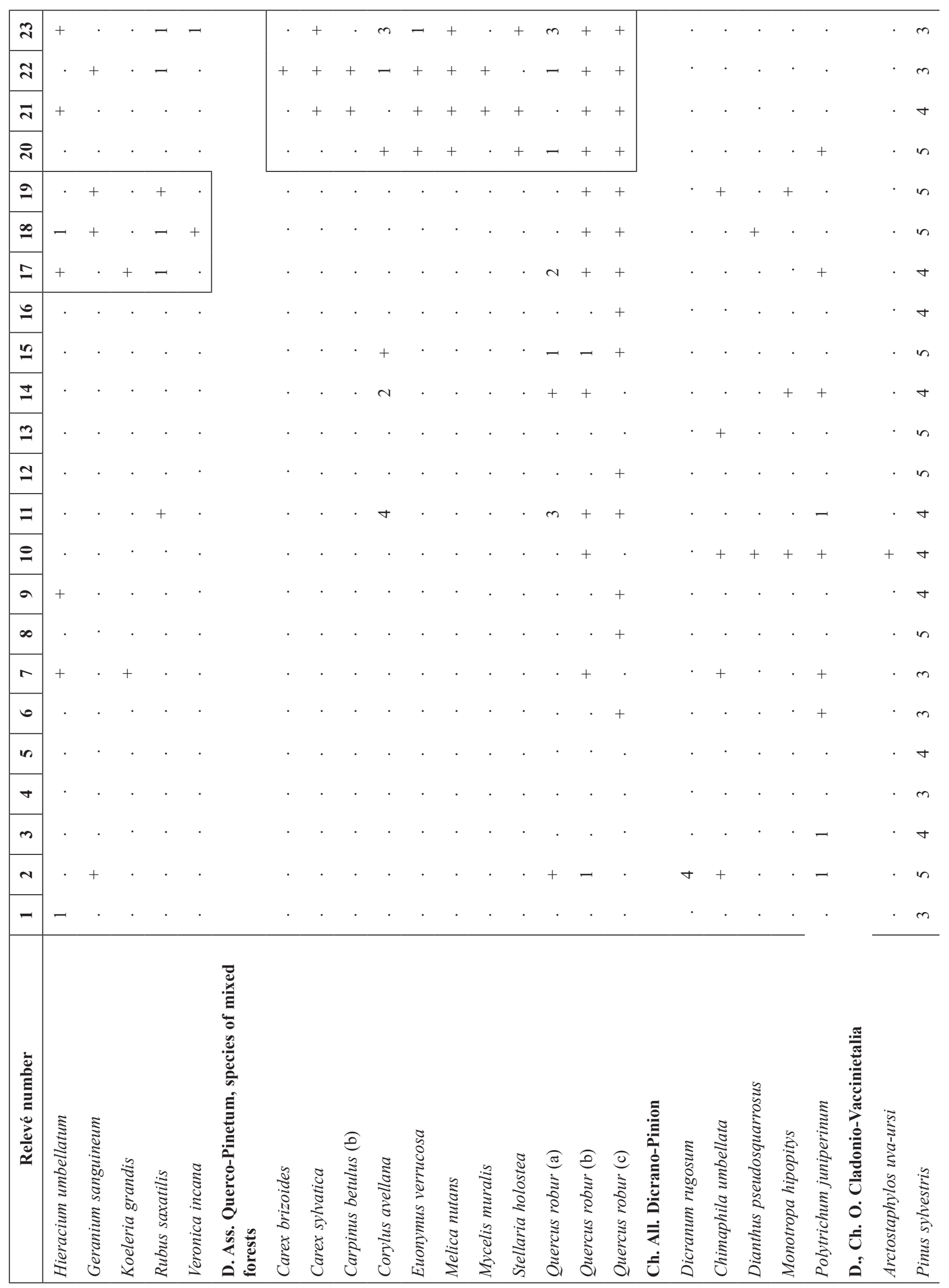




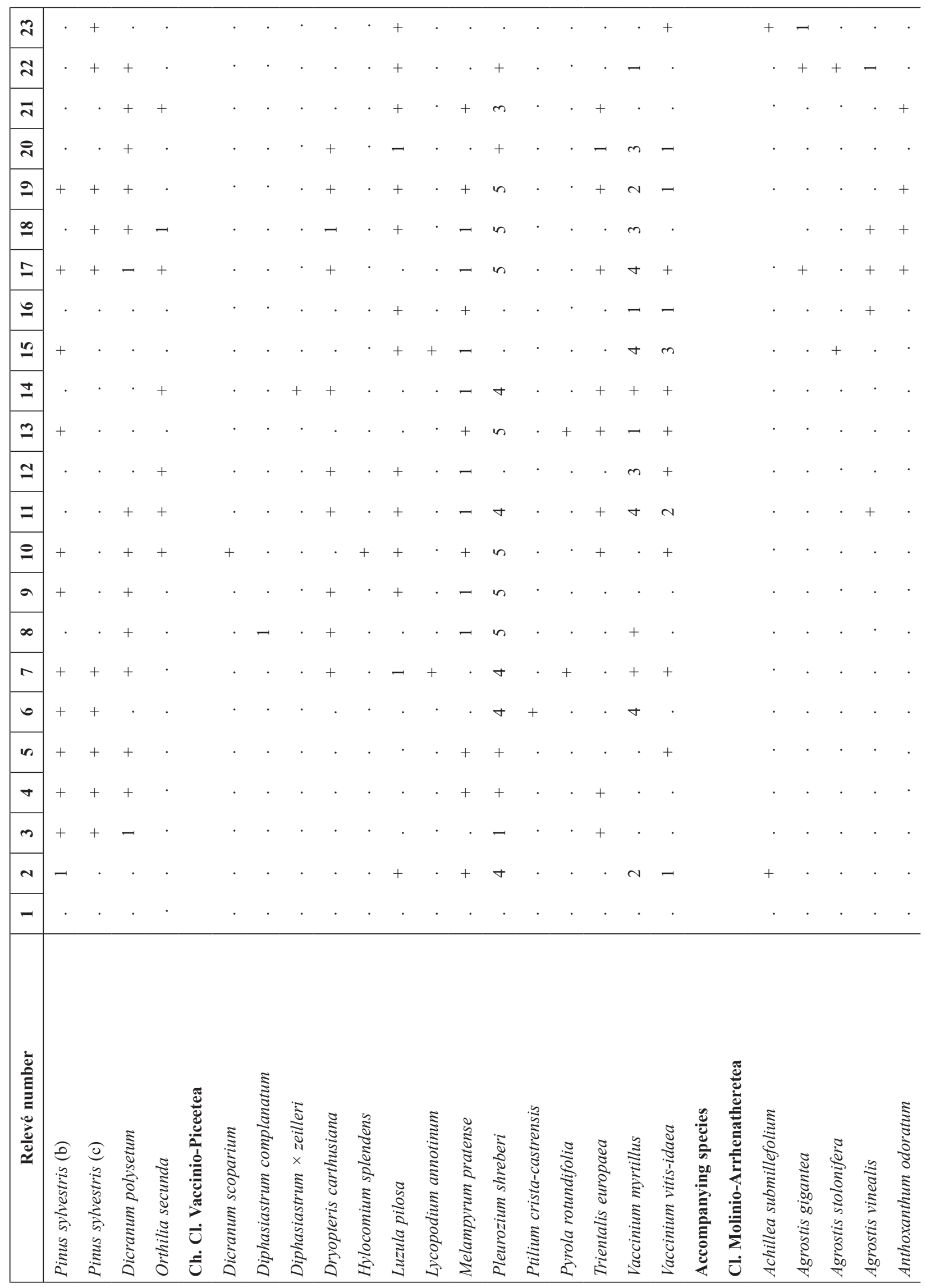




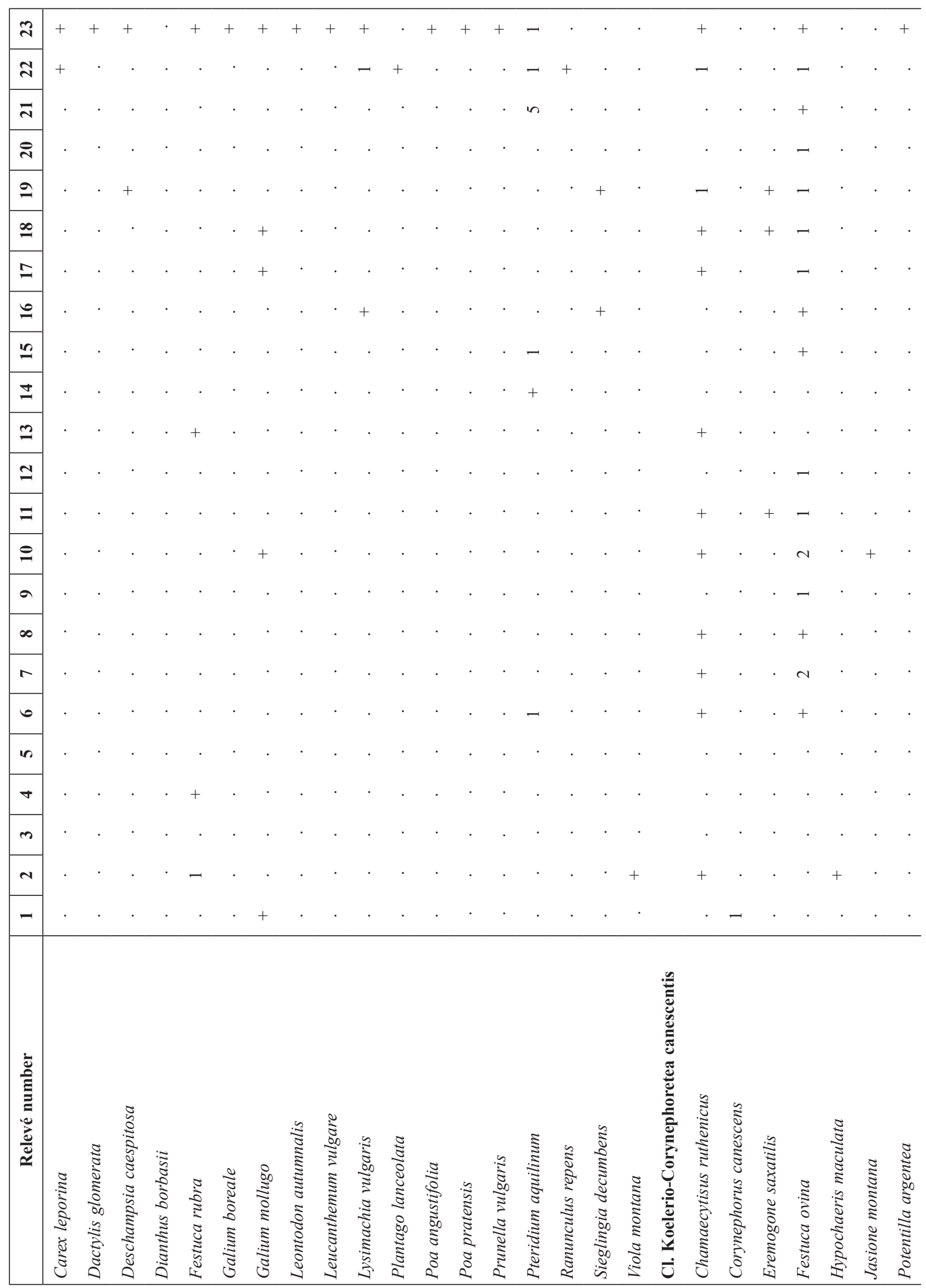




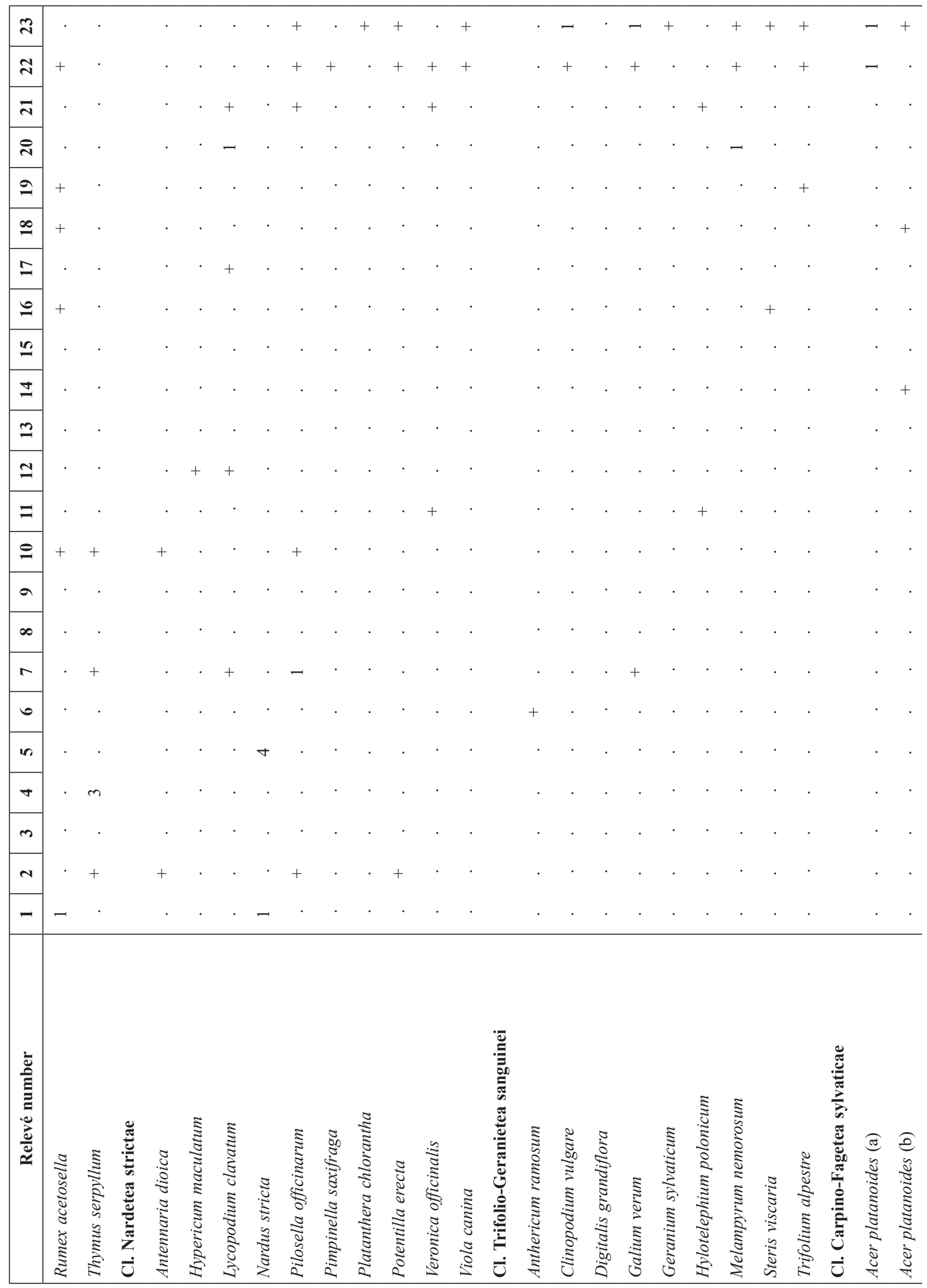




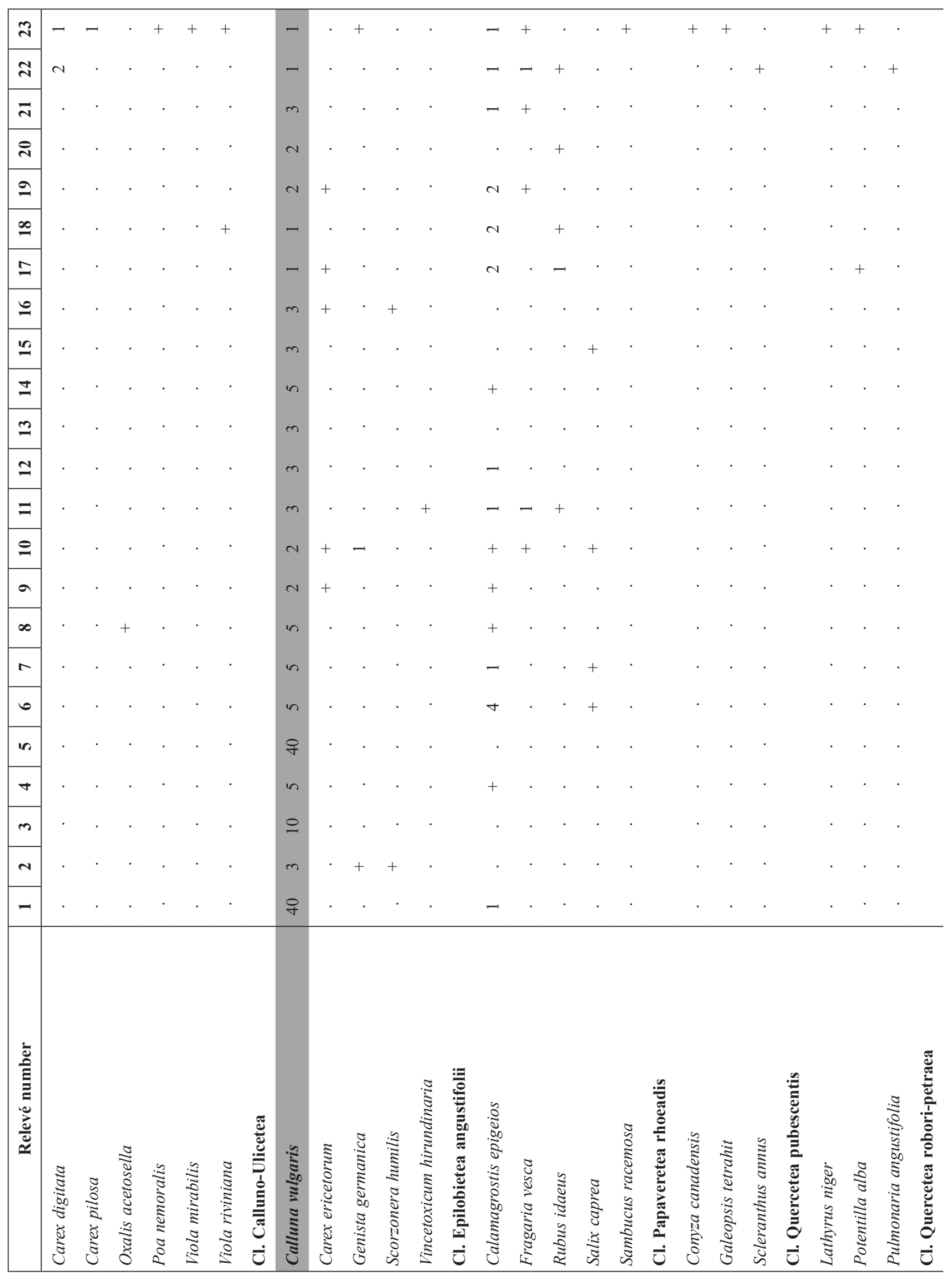




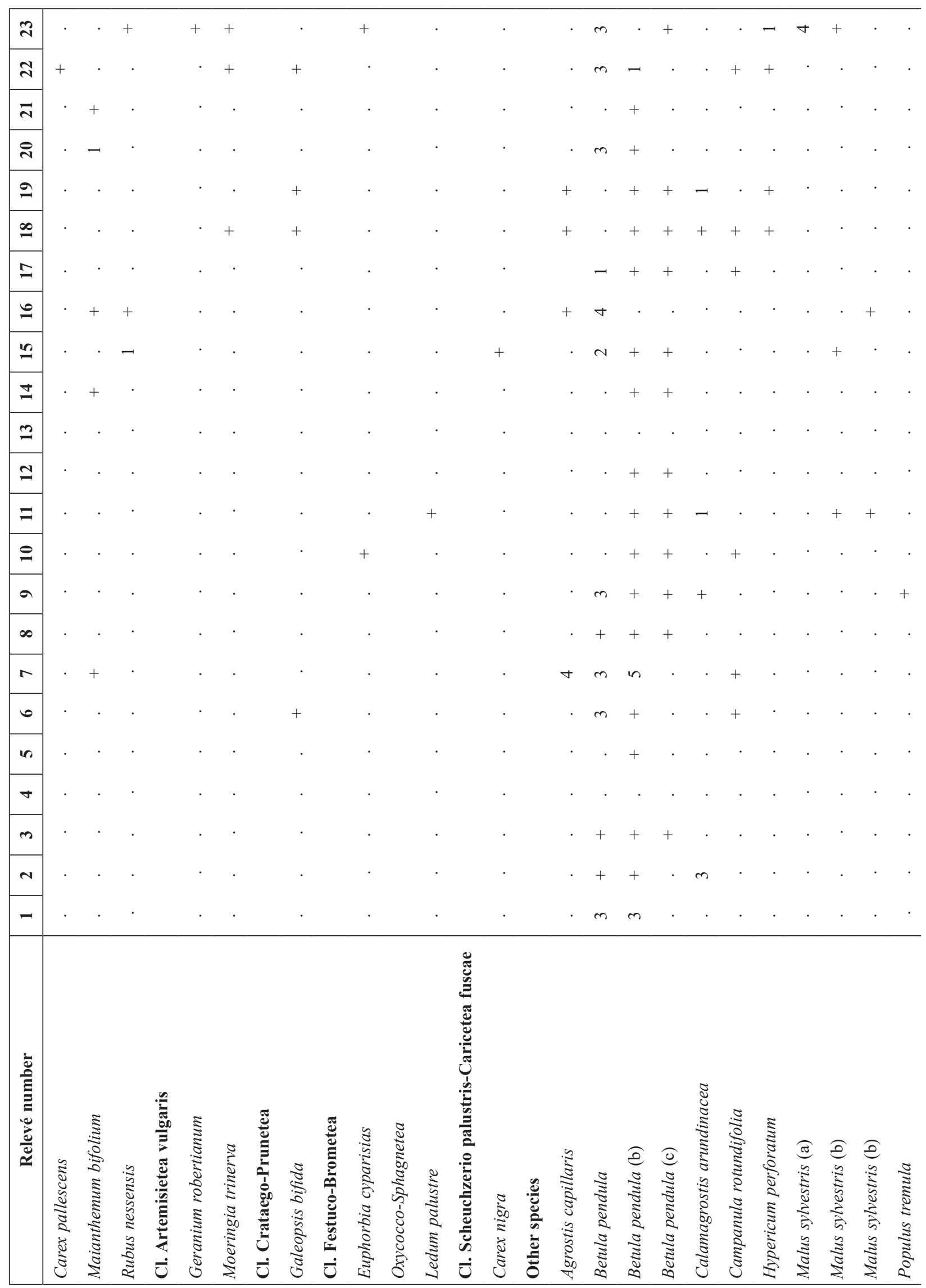



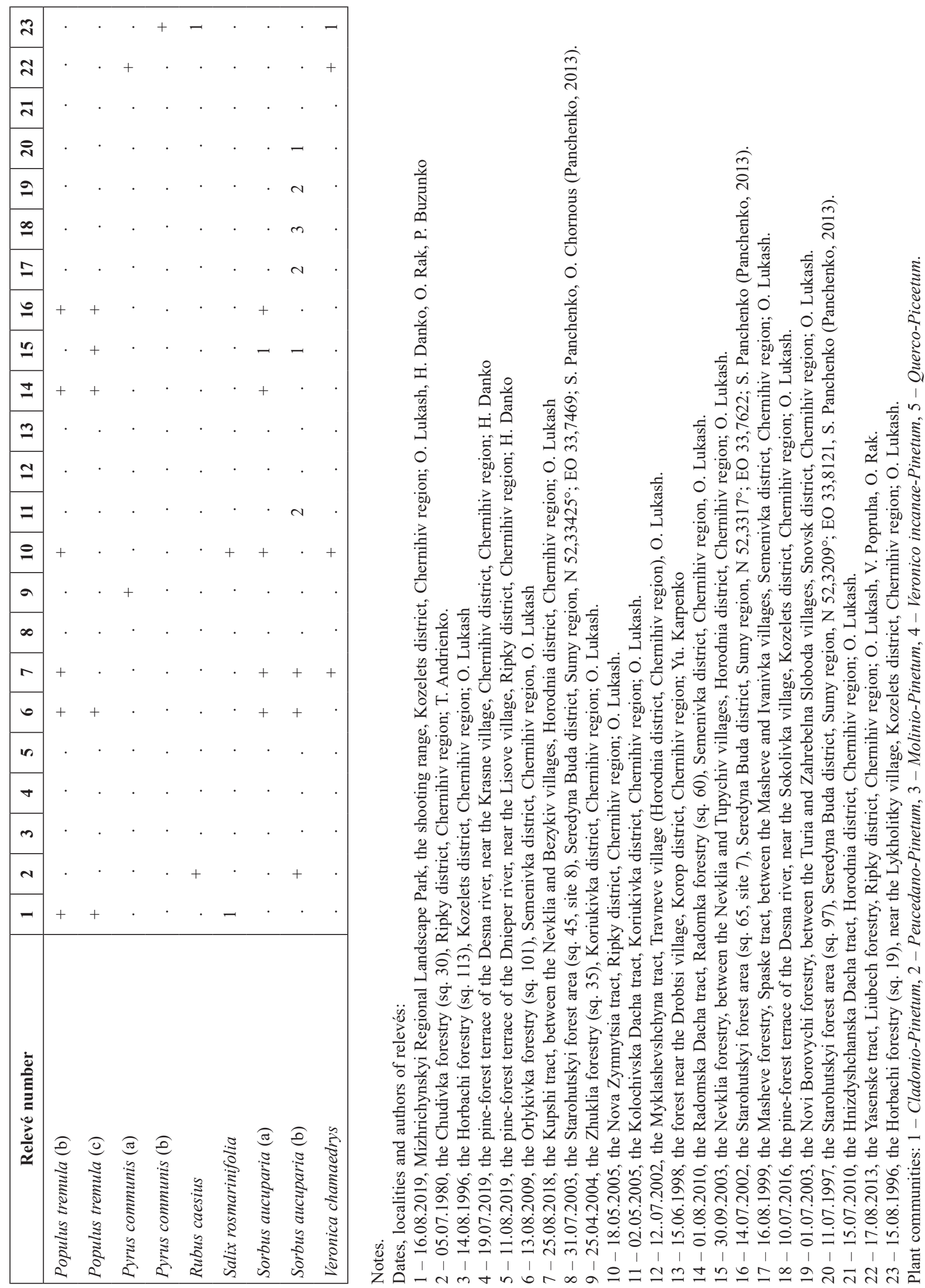
The ontogenetic structure of Calluna vulgaris in plant communities of pine forests of the Cladonio-Pinetum association was investigated. Two model cenopopulations of Calluna vulgaris (equal in area, ecological and phytocenotic conditions) were selected: in Right-bank Polesie - in the Rivne Nature Reserve (Table 4, relevé 1) and in Left-bank Polesie - in Mizhrichynskyi Regional Landscape Park (Table 5, relevé 1). The results of population studies are represented in Table 6 and Figure 2.

Table 6 shows that the density of the "right-bank" population is 118 individuals per $\mathrm{m}^{2}$. It has incomplete age spectrum (without seedlings and senile individuals) and is right-sided, with the peak on virginal and generative individuals (Fig. 2: I). The density of the "left-bank" population is 120 individuals per $\mathrm{m}^{2}$. It is also incomplete (without seedlings, juvenile and senile individuals) and rightsided with the peak on old generative individuals (Fig. 2: II). The difference in indexes of the ontogenetic status assessment of two populations indicates the following: the growth conditions for Calluna vulgaris in the Rivne Nature Reserve are more optimal and are followed by more active growth of plants and settling on new territories.

Table 6. Calluna vulgaris (L.) Hill. populations in Cladonio-Pinetum Juraszek 1927 communities of Ukrainian Polesie

\begin{tabular}{|l|c|c|c|c|c|c|c|c|c|c|c|c|c|}
\hline \multirow{2}{*}{ Population localization } & \multicolumn{7}{|c|}{ Average number of individuals/m ${ }^{2}$} & \multicolumn{5}{c|}{$\begin{array}{c}\text { Index of ontogenetic status } \\
\text { assessment }\end{array}$} \\
\cline { 2 - 14 } & $\mathbf{p}$ & $\mathbf{j}$ & $\mathbf{i m}$ & $\mathbf{v}$ & $\mathbf{g}_{1}$ & $\mathbf{g}_{2}$ & $\mathbf{g}_{3}$ & $\mathbf{s}$ & $\mathbf{\Sigma}$ & $\mathbf{I}_{\text {in }}$ & $\mathbf{I}_{\text {sen }}$ & $\mathbf{I}_{\text {gen }}$ & $\mathbf{I}_{\text {aet }}$ \\
\hline $\begin{array}{l}\text { Rivne Nature Reserve } \\
\text { (Right-bank Polesie) }\end{array}$ & 0 & 3 & 5 & 30 & 27 & 28 & 25 & 0 & 118 & 32.20 & 21.19 & 67.80 & 0.65 \\
\hline $\begin{array}{l}\text { Mizhrichynskyi Regional } \\
\text { Landscape Park (Left-bank } \\
\text { Polesie) }\end{array}$ & 0 & 0 & 2 & 29 & 25 & 24 & 40 & 0 & 120 & 25.83 & 33.33 & 40.83 & 1.29 \\
\hline
\end{tabular}

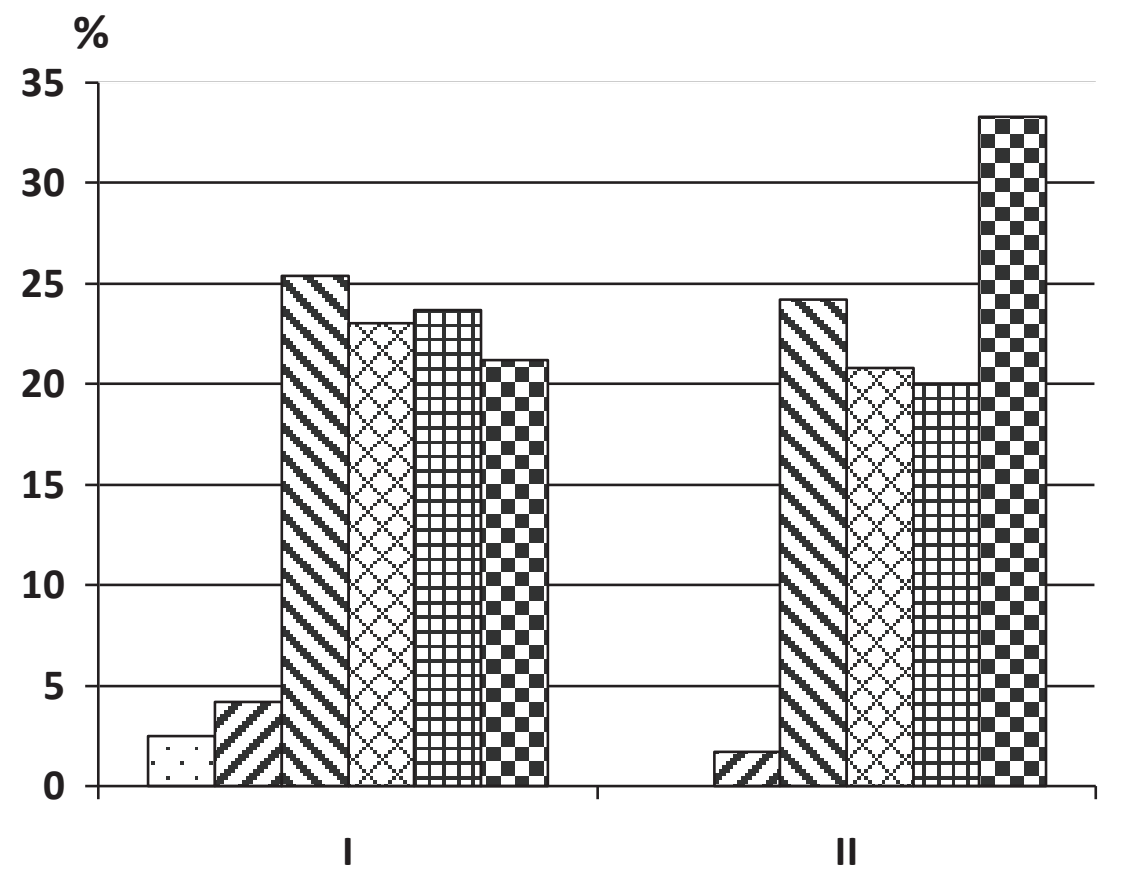

$\square j \nabla \mathrm{im} \nabla v \otimes g 1 \boxplus g 2 \boxminus g 3$

Figure 2. Age-related structure of the Calluna vulgaris populations in Rivne Nature Reserve (I) and Mizhrichynskyi Regional Landscape Park (II). Denotations: $\mathrm{j}$ - juvenile individuals, im - immature individuals, $v$ - virginile individuals; $\mathrm{g}_{1}-$ young generative individuals, $\mathrm{g}_{2}-$ mature generative individuals, $\mathrm{g}_{3}-$ old generative individuals 
The "western" population is younger $\left(\mathrm{I}_{\text {aet }}=0.65\right)$ than the "eastern" one, where the aging processes are prevailing $\left(\mathrm{I}_{\text {aet }}=1.29\right)$. To compare with, the aging of the Calluna vulgaris populations in the forest phytocenoses in the east of Left-bank Polesie (National Nature Park "DesnianskoStarohutskyi"), is more intensive: Index aetas $\left(\mathrm{I}_{\text {aet }}\right)$ is 1.72 (Kovalenko, 2015).

It should be noted that the syntaxonomical affiliation of the Calluna vulgaris forest habitats in Right-bank and Leftbank Polesie is different. In particular, rare, as for Polesie, communities of the Veronico incanae-Pinetum association (Table 5, relevés 17-19) occur only in Left-bank Polesie. Pine forests of the Vaccinio uliginosi-Pinetum association with Calluna vulgaris (Table 4, relevés 23-26) were described only in Right-bank Polesie. The communities of the Vaccinio uliginosi-Pinetum association are known only in National Nature Park "Desniansko-Starogutskyi" in Left-bank Polesie (Panchenko, 2013). Calluna vulgaris as well as Vaccinium uliginosum L. (diagnostic species of the Piceo-Vaccinienion uliginosi suballiance) are absent in all 10 relevés of this association. The European boreomontane spruce forests of the Piceion excelsae class (Table 4, relevés 27-29) occur only in Right-bank Polesie. The phytocenoses are represented by the Sphagno girgensohnii-Piceetum association. The projective coverage of Calluna vulgaris does not exceed $1 \%$.
According to the results of the research, the map of the Calluna vulgaris distribution in Ukrainian Polesie biotopes was created (Fig. 3).

We compared the frequency of the Calluna vulgaris constancy in plant communities of Right-bank and Leftbank Polesie. The results of the comparison are shown in Table 7.

Undoubtedly, Calluna vulgaris has the maximum constancy frequency in the phytocenoses of the CallunoUlicetea and the Nardetea strictae classes, where it is a characteristic or differentiating (diagnostic) species. The Calluna vulgaris high constancy is observed in the plant communities of the Ledo-Sphagnetum magellanici association. The Calluna vulgaris occurrence in pine forests of Right-bank Polesie is slightly higher (than in Left-bank Polesie). The spruce forests of the Sphagno girgensohniiPiceetum association are rare biotopes for Calluna vulgaris. It should be noted that Calluna vulgaris does not grow in other spruce forests, which have an "island" distribution in Polesie. Rather stable position and high constancy of Calluna vulgaris in different phytocenoses of Ukrainian Polesie is a consequence of high drought and frost resistance of this species in combination with plasticity of its morphological structure (Grubb, 1992).

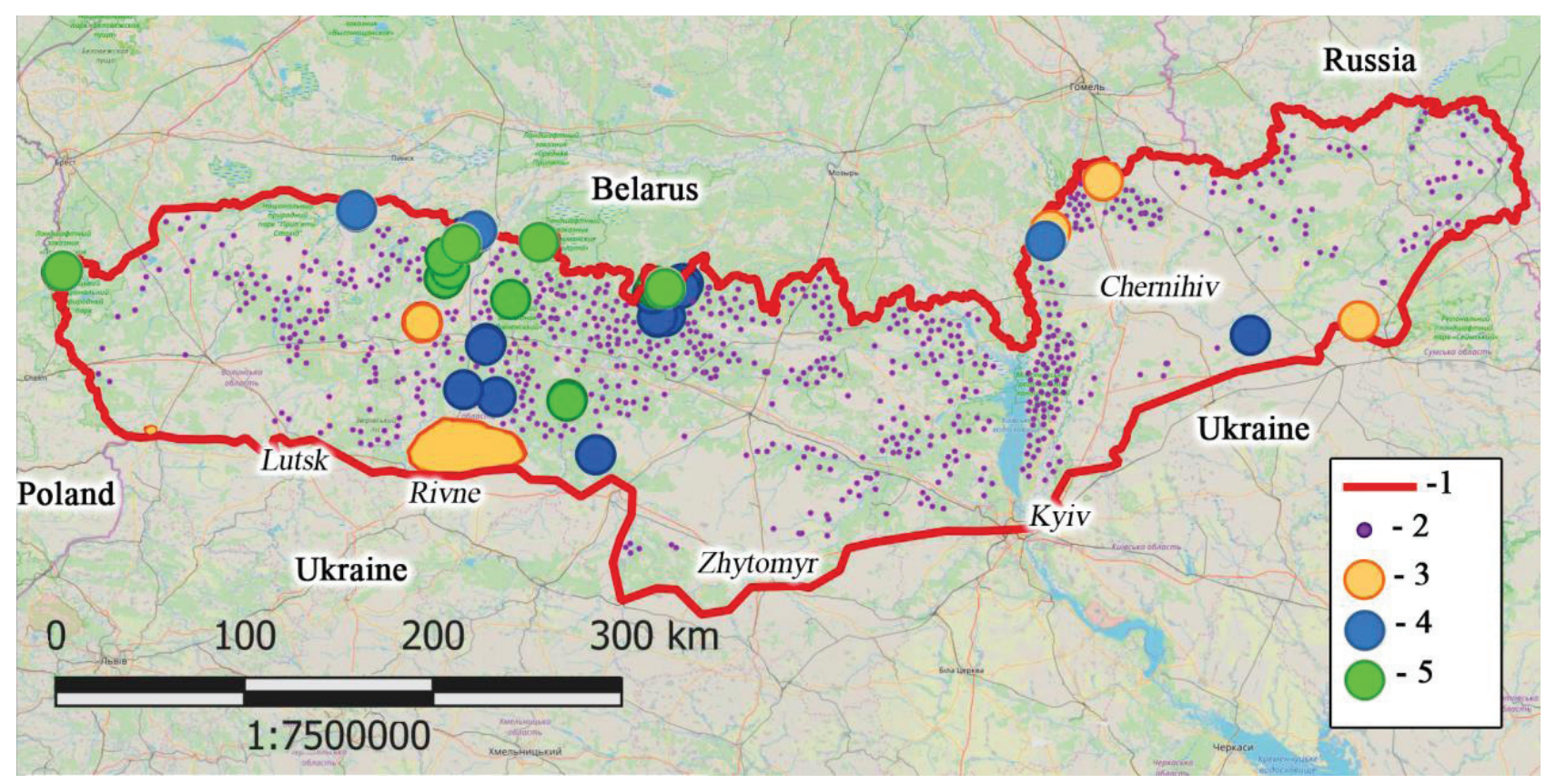

Figure 3. Distribution of Calluna vulgaris (L.) Hill. in the biotopes of Ukrainian Polesie. Map symbols: 1 - border of Ukrainian Polesie, 2 - temperate and subboreal pine forests on nutrient-poor acidic sandy soils (the Vaccinio-Piceetea class), 3 boreo-temperate heathlands of the Calluno-Uliceteaclass, 4 - boreo-temperate heathlands of the Nardetea strictae class, 5 - dwarf-shrub, sedge and peat-moss ombrotrophic raised bogs on extremely acidic soils (the Oxycocco-Sphagnetea class) 
Table 7. Constancy of Calluna vulgaris (L.) Hill. in plant communities of Ukrainian Polesie

\begin{tabular}{|l|c|c|c|c|c|c|}
\hline \multirow{2}{*}{ Association } & \multicolumn{3}{|c|}{ Right-bank Polesie } & \multicolumn{3}{c|}{ Lef t-bank Polesie } \\
\cline { 2 - 7 } & $\begin{array}{c}\text { Total } \\
\text { number } \\
\text { of relevés } \\
\text { analyzed }\end{array}$ & $\begin{array}{c}\text { Number } \\
\text { of relevés } \\
\text { with } \\
\text { Calluna } \\
\text { vulgaris }\end{array}$ & $\begin{array}{c}\text { Nonstancy, } \\
\text { \% } \\
\text { number } \\
\text { of relevés } \\
\text { analyzed }\end{array}$ & $\begin{array}{c}\text { Number } \\
\text { relevés } \\
\text { Calluna } \\
\text { vulgaris }\end{array}$ & $\begin{array}{c}\text { Constancy, } \\
\%\end{array}$ \\
\hline Calluno-Genistetum pilosae & 3 & 3 & 100 & 0 & 0 & - \\
\hline Scabioso canescentis-Genistetum & 0 & 0 & - & 5 & 5 & 100 \\
\hline Calluno-Nardetum strictae & 17 & 17 & 100 & 4 & 4 & 100 \\
\hline Ledo-Sphagnetum magellanici & 41 & 38 & 92.7 & 0 & 0 & - \\
\hline Cladonio-Pinetum & 67 & 43 & 64.2 & 63 & 36 & 57.1 \\
\hline Peucedano-Pinetum & 82 & 63 & 76.8 & 93 & 68 & 73.1 \\
\hline Veronico incanae-Pinetum & 0 & 0 & - & 43 & 38 & 65.1 \\
\hline Molinio-Pinetum & 57 & 41 & 71.9 & 36 & 25 & 69.4 \\
\hline Querco-Piceetum & 45 & 18 & 40.0 & 48 & 19 & 39.6 \\
\hline Vaccinio uliginosi-Pinetum & 36 & 17 & 47.2 & 10 & 0 & 0 \\
\hline Sphagno girgensohnii-Piceetum & 43 & 7 & 16.3 & 0 & 0 \\
\hline
\end{tabular}

\section{Conclusions}

The heathlands are rare ecosystems in Ukrainian Polesie. Their vegetation is represented by the plant communities of the Calluno-Ulicetea and the Nardetea strictae classes. They are forming in greatly anthropogenically disturbed areas (e.g., areas after wildfires) or in the areas with intensive human activities (at shooting ranges first of all). The cenoses of the Calluno-Genistetum pilosae association, which are present only in Right-bank Polesie, have larger areas (up to $20 \mathrm{~km}^{2}$ ). Other heather communities are smaller in size; they usually occupy the area of several hectares (the Calluno-Nardetum strictae association) or are represented by small patches in landscape of pine forests (less frequently in mixed forests) on upland terraces of rivers (the Scabioso canescentis-Genistetum association).

A significant participation (with the projective cover of up to $80 \%$ ) and high constancy $(92.7 \%)$ in oligotrophic swamps the phytocenoses of the Ledo-Sphagnetum magellanici association is a characteristic feature of the Calluna vulgaris distribution in Right-bank Polesie of Ukraine.

The middle-aged pine and birch-pine forests of the $D i$ crano-Pinenion suballiance are the most common habitats of Calluna vulgaris in Ukrainian Polesie (mostly, with its projective cover of less than $1 \%$ ). The phytocenoses of the
Veronico incanae-Pinetum association is the habitat of Calluna vulgaris only in Left-bank Polessie. Calluna vulgaris has the lowest constancy (57.1\%) in the Cladonio-Pinetum association of Left-bank Polesie and the highest constancy (76.8\%) in the Peucedano-Pinetum association of Rightbank Polesie. Calluna vulgaris constancy does not exceed $40 \%$ in the oak-pine forests of the Querco-Piceetum association. The syntaxonomic difference of the Calluna vulgaris forest habitats in Right-bank Polesie and Left-bank Polesie is primarily revealed at the Piceo-Vaccinienion uliginosi suballiance level and the Piceetalia excelsae order. The spruce forests of the Sphagno girgensohnii-Piceetum association are rare biotopes for Calluna vulgaris.

The Calluna vulgaris populations within the forest plant communities of the Cladonio-Pinetum association are characterized by a similar ontogenetic structure: they are incomplete. There are no sprouts, vegetative reproduction prevails, and age spectrum maximum falls on generative individuals. However, the Calluna vulgaris cenotic population in Right-bank Polesie of Ukraine is characterized by a greater activity of plants growth and spreading on new territories, while aging prevails in the "left bank" population.

In general, the environmental conditions of Right-bank Polesie of Ukraine are more favorable to Calluna vulgaris, than the conditions in Left-bank Polesie. 


\section{References}

Adamska E., Deptuła M., Filbrandt-Czaja A., Kamiński D., Lewandowska-Czarnecka, Nienartowicz A. \& Sewerniak P., 2015, Heathlands and Associated Communities in Kujawy and Pomerania: Management, Treatment and Conservation. Towarzystwo Naukowe w Toruniu, Torun.

Andrienko T. L., Onischenko V. A., Priadko O. I., Panchenko S. M., Arap R. Ya., Kinischuk V. V., Lukash O. V., Karpenko Yu. O., Virchenko V. M. \& Chornous O. P., 2006, Phytodiversity of Ukrainian Polesie and its conservation. T. L. Andrienko (ed.), Phytosociocentre, Kyiv.

Bulohov A.D. \& Solomeshch A.I., 2003, Syntaxonomy of forests of Russian South Nechernozemie, Publishing house of Bryansk State University, Bryansk.

Chojnacka J., Cyzman W., Nienartowicz A. \& Deptuła M., 2010a, Variability of the structure and directions in the development of heaths and psammophilous grasslands within the artillery range near the city of Torun. Ecological Questions 12: 87-126.

Chojnacka J., Cyzman W., Nienartowicz A. \& Deptuła M., 2010b, Comparison of heath communities from the Nardo-Callunetea class from the Torun Basin and other regions of Poland. Ecological Questions 12: 87-126.

Fagúndez J., 2013, Heathlands confronting global change: drivers of biodiversity loss from past to future scenarios. Annals of Botany 111: 151-172.

Fijałkowski D., Andrienko T., Onyszenko W., Święs F. \& Urban D., 2002, Ekosystemy lądowe-leśne, kserotermiczne i synantropijne Polesia (Flora i fitocenozy) [Terrestrial and forest, xerothermic, and synanthropic ecosystems of Polesie (Flora and phytocoenoses)]. Acta Agrophysica 66: 147-177.

Grub P.J., 1992, A positive distrust in simplicity: lessons from plant defenses and from competition among plants and among animals. Journal of Ecology 80: 585-610.

Korchagin A.A. \& Lavrenko E.M., 2012, Field geobotany. Methodical guidance. E. M. Lavrenko (ed.), PH Ozon. ru, Moscow.
Kovalenko I.N., 2015, Populations of Calluna vulgaris (L.) Hull. in the forest phytocenoses of the National Nature Park "Desniansko-Starogutsky" (Sumy region, Ukraine). Chornomorsky Botanical Journal 11(4): 438448.

Lukash O., Miroshnyk I. Yakovenko O. \& Strilets S., 2019, The vegetation of the cretaceous outcrops of NovhorodSiverskyi Polesie loess "islands" (Ukraine) and the new locality of Gentiana cruciata L. Ecological Questions 30(1): 21-33.

Lukash O., Yakovenko O. \& Miroshnyk I., 2018, The mechanical degradation of the land surface and the present state of the loess "islands" plant cover of Chernihiv Polesie (Ukraine). Ecological Questions 29(4): 23-34.

Matuszkiewicz W., 2019, Przewodnik do oznaczania zbiorowisk roślinnych Polski [Guide to the determination of Polish plant communities]. Wydawnictwo Naukowe PWN, Warszawa.

Mucina L., Büultmann H., Dierßen K., Theurillat J.-P., Raus T., Čarni A., Šumberová K., Willner W., Dengler J., García R. G., Chytrý M., Hájek M., Di Pietro R., Iakushenko D., Pallas J., Daniëls F. J. A., Bergmeier E., Guerra A. S., Ermakov N., Valachovič M., Schaminće J. H. J., Lysenko T., Didukh Y. P., Pignatti S., Rodwell J. S., Capelo J., Weber H. E., Solomeshch A., Dimopoulos P., Aguiar C., Hennekens S. M. \& Tichý L., 2016, Vegetation of Europe: hierarchical floristic classification system of vascular plant, bryophyte, lichen, and algal communities. Applied Vegetation Science 19(S1): 3-264. https://onlinelibrary.wiley.com/doi/ epdf/10.1111/avsc. 12257

Panchenko S.M, 2013, Forest vegetation of the National Natural Park "Desniansko-Starogutsky", University Book, Sumy.

Rabotnov T.A., 1964, Determination of age composition of species populations in natural plant communities. Field geobotany. Methodical guidance. Vol.3. E. M. Lavrenko (ed.), Nauka, Moscow, pp. 132-145. 